\title{
Displacements and stress distribution in DO Run IIb stave due to CTE mismatches
}

\author{
Giobatta Lanfranco, James Fast \\ Fermi National Accelerator Laboratory \\ Particle Physics Division / Silicon Engineering Group - Mechanical Dep. \\ e-mail: giobatta@fnal.gov,jfast@fnal.gov
}

24 July 2001

\section{Abstract}

A possible D0 Run IIb stave design currently under study is characterized by an outer carbon fiber stiffening shell with the silicon detectors mounted internally and a single central cooling line running between them; in this paper the stave will be analyzed for thermal compatibility since the different coefficient of thermal expansion in the materials may cause unpredictable stresses and strains in the structure. A simplified stave section has been modeled with finite elements for different materials configurations and the vertical and longitudinal displacements induced by the thermal gradient, together with the related stresses, have been computed. Finally, once selected the most suitable material combination, a more realistic model has been created in order to study the influence of the hybrid location along the ladders.

\section{Stave layout}

The stave is constructed from $200 \mathrm{~mm}$ long ladders consisting of two silicon sensors, $37.2 \mathrm{~mm}$ wide by $100 \mathrm{~mm}$ long, glued together end-to-end with a hybrid, $35 \mathrm{~mm}$ wide and $60 \mathrm{~mm}$ long, glued to the silicon, centered on the sensor to sensor joint. Six of these ladder structures are joined end-to-end to form a $1.2 \mathrm{~m}$ long chain and two chains are attached to opposite sides of the stave core and cooling lines. Each hybrid has 10 SVX chips mounted on it for a total heat load of $5 \mathrm{~W}$ per hybrid. Because of the way the silicon sensors are ganged together, the hybrids may be spaced facing each other or in alternate order; these two configurations with their asymmetry may induce higher thermal gradient related displacements and have been investigated at the end of the present paper. 
Fermi National Accelerator Laboratory

Giobatta Lanfranco Silicon Engineering Group - Mechanical Dep.

The $1.2 \mathrm{~m}$ long silicon chains are bonded to either side of the core with axial strip readout on one side and small angle stereo strip readout on the other. Finally, two composite C-channels are attached around the silicon to form a box structure that provides most of the stave stiffness. The stave length is 48 " $(1.22 \mathrm{~m})$ with support provided at the center and the two ends. The silicon supporting blocks are equally spaced and are $2 \mathrm{~mm}$ high x $4 \mathrm{~mm}$ long x $8 \mathrm{~mm}$ wide.

Figure 1 shows a cross-section of the stave. Table 1 summarizes the stave constituent materials, their thickness and their thermal conductivities.

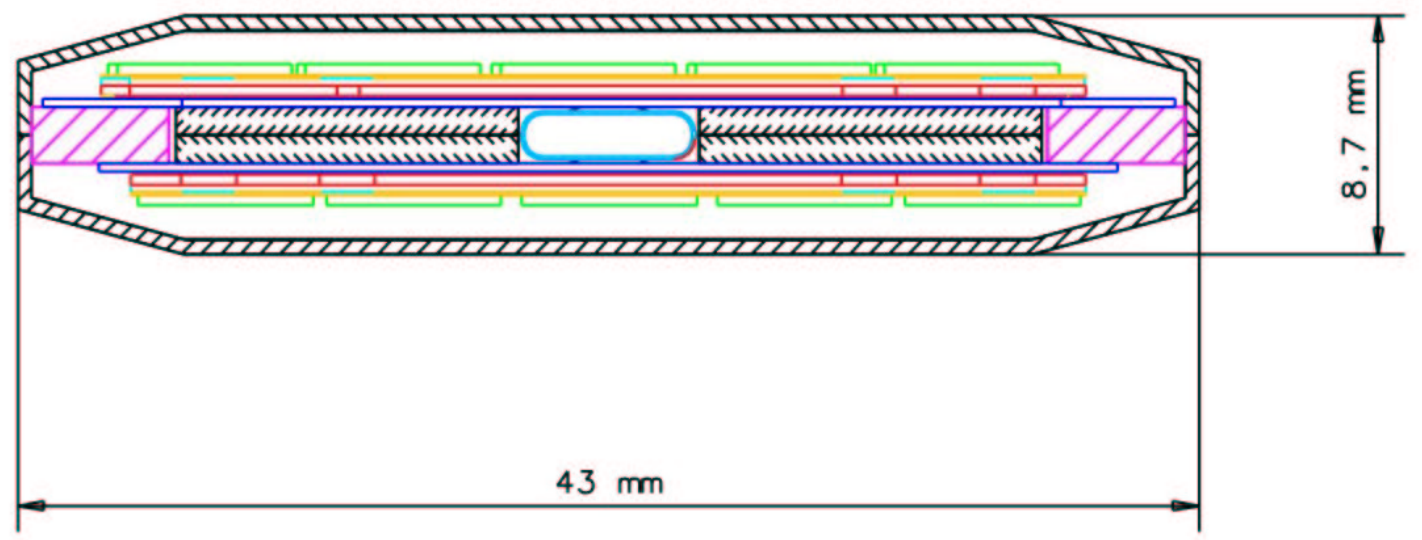

Figure 1 - The ladder section

\begin{tabular}{ccccc}
\hline Component & material & $\begin{array}{c}\text { Thickness } \\
{[\mu \mathrm{m}]}\end{array}$ & $\begin{array}{c}\text { Thermal } \\
\text { conductivity }[\mathbf{W} / \mathbf{m K}]\end{array}$ & $\begin{array}{c}\text { Color code in } \\
\text { Figure } \mathbf{1}\end{array}$ \\
\hline Support shell & investigated & 500 & - & black \\
\hline Sensor & Silicon & 380 & 124 & blue \\
\hline Chip & Silicon & 300 & 124 & green \\
\hline Glue & Epoxy & 50 & 1 & not depicted \\
\hline Tubing & PEEK & 100 & 0.25 & cyan \\
\hline HDI & Kapton & 100 & 0.2 & gold \\
\hline Substrate & BeO & 500 & 250 & red \\
\hline Heat spreader & investigated & 100 & - & not depicted \\
\hline Core & Rohacell & 2000 & $\sim 0$ & black (short hatch) \\
\hline Support block & ceramic & 2000 & 1.46 & magenta \\
\hline
\end{tabular}

Table 1 - Thermal conductivities and thickness of the stave constituent materials 
Fermi National Accelerator Laboratory

Giobatta Lanfranco Silicon Engineering Group - Mechanical Dep.

\section{Finite element models}

The model analyzed is constituted by a $\mathrm{C}$ shaped shell supporting a silicon sensor glued to a heat spreader. The material of the shell structure and of the heat spreader, as well as the number of the supports, have been varied so to have the following six configurations:

\begin{tabular}{|c|c|c|c|c|}
\hline Case & Shell & Heat Spreader & Core & Supports \\
\hline 1 & K139 + Boron prepreg ${ }^{1}$ & hypothetical & - & 4 \\
\hline 2 & $\mathrm{~K} 139+$ Boron prepreg $^{1}$ & kapton & - & 4 \\
\hline 3 & $\mathrm{~K} 139+$ Boron prepreg $^{1}$ & $\mathrm{~K} 13 \mathrm{D} 2 \mathrm{U}^{2}$ & - & 4 \\
\hline 4 & $\mathrm{~K} 139\left[90^{\circ}-0^{\circ}\right]^{3}$ & $\mathrm{~K} 13 \mathrm{D} 2 \mathrm{U}^{2}$ & - & 4 \\
\hline 5 & $\mathrm{~K} 139\left[90^{\circ}-0^{\circ}\right]^{3}$ & $\mathrm{~K} 13 \mathrm{D} 2 \mathrm{U}^{2}$ & - & 6 \\
\hline 6 & $\mathrm{~K} 139\left[90^{\circ}-0^{\circ}\right]^{3}$ & $\mathrm{~K} 13 \mathrm{D} 2 \mathrm{U}^{2}$ & Rohacell & 6 \\
\hline
\end{tabular}

Table 2 - Finite element models studied

The K139 + Boron prepreg closely matches the coefficient of thermal expansion (CTE) of silicon along the longitudinal direction $(\mathrm{z})$, so that no stresses are introduced in the sensor. The $\mathrm{K} 139\left[90^{\circ}-0^{\circ}\right]$ offers instead higher mechanical properties and presents no significant longitudinal elongation ${ }^{4}$, with the advantage of an easier stave anchoring.

The heat spreader, although the misleading name, has the main function to help with assembling and handling the silicon sensor chain; that explains the choice of testing kapton although its poor thermal conductivity $(K)$. K13D2U shows a similar low inter-laminar thermal conductivity, since in the through-plane direction the heat transfer is mainly relying on the resin. However a K13D2U heat spreader is preferable to kapton for its better mechanical properties and for the fact that the higher transversal and longitudinal values of $\mathrm{K}$ facilitate spreading the heat to regions at lower heat density giving a smoother temperature gradient distribution.

The hypothetical material in the heat spreader of case 1 is a substance perfectly matching CTE and elastic modulus of silicon.

\footnotetext{
${ }^{1}$ See Table 3.

${ }^{2}$ See Table 5 .

${ }^{3}$ See Table 4.

${ }^{4}$ Because of its virtually zero CTE.
} 
Fermi National Accelerator Laboratory

Giobatta Lanfranco Silicon Engineering Group - Mechanical Dep.

The supports are small ceramic blocks and are thought perfectly fixed to the Silicon. No glue has been modeled except at the interface between silicon and heat spreader and between heat spreader and Rohacell (configuration 6).

From Table 3 to Table 5 the properties of the carbon fiber laminates are shown. In Table 6 the Young's modulus and the CTE of the materials used are given.

Boron 5505/4 + k139-1515-70/30

\begin{tabular}{cccc}
\hline Ply \# & Lamina Type & Thickness & Angle \\
\hline 1 & k139-1515-70/30 & 55 & 54 \\
2 & Boron 5505/4 & 135 & 0 \\
3 & k139-1515-70/30 & 55 & -54 \\
4 & k139-1515-70/30 & 55 & -54 \\
5 & Boron 5505/4 & 135 & 0 \\
6 & k139-1515-70/30 & 55 & 54
\end{tabular}

\begin{tabular}{||c|c||}
\hline Ex (pa) & $106.2 \mathrm{GPa}$ \\
Ey (pa) & $77.5 \mathrm{GPa}$ \\
Ez (pa) & $15.7 \mathrm{GPa}$ \\
Gxy (pa) & $46.9 \mathrm{GPa}$ \\
Gxz (pa) & $0.8 \mathrm{GPa}$ \\
Gyz (pa) & $0.9 \mathrm{GPa}$ \\
NUxy & 0.49 \\
NUyx & 0.36 \\
NUxz & 0.14 \\
NUzx & 0.02 \\
NUyz & 0.31 \\
NUzy & 0.06 \\
CTEx & $2.50 \mathrm{E}-06 \mathrm{~mm} / \mathrm{mm}^{\circ}{ }^{\circ} \mathrm{C}$ \\
CTEy & $1.03 \mathrm{E}-07 \mathrm{~mm} / \mathrm{mm} /{ }^{\circ} \mathrm{C}$ \\
CTEz & $3.31 \mathrm{E}-05 \mathrm{~mm} / \mathrm{mm} /{ }^{\circ} \mathrm{C}$ \\
Density & $1.875 \mathrm{~g} / \mathrm{cm} 3$ \\
Thickness & $490 \mathrm{Micron}$ \\
\hline \hline
\end{tabular}

Table 3 - Lay-up and properties of the laminate containingK139 and boron prepreg 
Fermi National Accelerator Laboratory

Giobatta Lanfranco Silicon Engineering Group - Mechanical Dep.

$\mathrm{K} 139\left[90^{\circ}-0^{\circ}\right]$

\begin{tabular}{|c|c|c|c|c|}
\hline \multirow{2}{*}{$\begin{array}{c}\text { \# Plies } \\
7\end{array}$} & \multirow{2}{*}{$\begin{array}{c}\text { Fiber type } \\
\text { Mitsubishi K139 }\end{array}$} & \multicolumn{3}{|c|}{ Resin Type Resin to Fiber ratio Thickne } \\
\hline & & EX 1515 & $70 / 30$ & 7 \\
\hline & & Ex (pa) & 185 & $\mathrm{GPa}$ \\
\hline & & Ey (pa) & 245 & $\mathrm{GPa}$ \\
\hline & & Ez (pa) & 8.4 & $\mathrm{GPa}$ \\
\hline & & Gxy (pa) & 2.2 & $\mathrm{GPa}$ \\
\hline & & Gxz (pa) & 1.7 & $\mathrm{GPa}$ \\
\hline & & Gyz (pa) & 1.8 & $\mathrm{GPa}$ \\
\hline & & NUxy & $7.7 \times 10^{-3}$ & \\
\hline & & NUyx & $1.02 \times 10^{-2}$ & \\
\hline & & NUxz & 0.53 & \\
\hline & & NUzx & $2.4 \times 10^{-2}$ & \\
\hline & & NUyz & 0.52 & \\
\hline & & NUzy & $1.8 \times 10^{-2}$ & \\
\hline & & CTEX & $-7.5 \times 10^{-7}$ & $\mathrm{~mm} / \mathrm{mm} /{ }^{\circ} \mathrm{C}$ \\
\hline & & CTEy & $-9.25 \times 10^{-7}$ & $\mathrm{~mm} / \mathrm{mm} /{ }^{\circ} \mathrm{C}$ \\
\hline & & CTEz & $3.41 \times 10^{-5}$ & $\mathrm{~mm} / \mathrm{mm} /{ }^{\circ} \mathrm{C}$ \\
\hline & & Density & 1.714 & $\mathrm{~g} / \mathrm{cm}^{3}$ \\
\hline & & Thickness & 525 & $\mu \mathrm{m}$ \\
\hline
\end{tabular}

Table 4 - Lay-up and properties of the laminate containing $K 139\left[90^{\circ}-0^{\circ}\right]$ 
Fermi National Accelerator Laboratory

Giobatta Lanfranco Silicon Engineering Group - Mechanical Dep.

K13D2U

\begin{tabular}{|c|c|c|c|c|}
\hline \# Plies & Fiber type & Resin Type & Resin to Fiber ratio Thickness [ $\mu \mathrm{m}$ ] & Angle Layout \\
\hline 3 & Mitsubishi K13D2U & EX 1515 & $70 / 30$ & $90^{\circ}-0^{\circ}-90^{\circ}$ \\
\hline
\end{tabular}

\begin{tabular}{||c|cl||}
\hline Ex (pa) & 176 & $\mathrm{GPa}$ \\
Ey (pa) & 346 & $\mathrm{GPa}$ \\
Ez (pa) & 8.4 & $\mathrm{GPa}$ \\
Gxy (pa) & 2.14 & $\mathrm{GPa}$ \\
Gxz (pa) & 1.59 & $\mathrm{GPa}$ \\
Gyz (pa) & 1.86 & $\mathrm{GPa}$ \\
NUxy & $5.48 \times 10^{-3}$ & \\
NUyx & $1.079 \times 10^{-2}$ \\
NUxz & 0.55 & \\
NUzx & $2.625 \times 10^{-2}$ \\
NUyz & 0.52 & \\
NUzy & $1.28 \times 10^{-2}$ & \\
CTEx & $-5 \times 10^{-7}$ & $\mathrm{~mm} / \mathrm{mm} /{ }^{\circ} \mathrm{C}$ \\
CTEy & $-8.81 \times 10^{-7}$ & $\mathrm{~mm} / \mathrm{mm} /{ }^{\circ} \mathrm{C}$ \\
CTEz & $3.46 \times 10^{-5}$ & $\mathrm{~mm} / \mathrm{mm} /{ }^{\circ} \mathrm{C}$ \\
Kx & 147.5 & $\mathrm{~W} / \mathrm{m} / \mathrm{K}$ \\
Ky & 295 & $\mathrm{~W} / \mathrm{m} / \mathrm{K}$ \\
Kz & 0.3 & $\mathrm{~W} / \mathrm{m} / \mathrm{K}$ \\
Density & 1.736 & $\mathrm{~g} / \mathrm{cm}{ }^{3}$ \\
Thickness & 99 & $\mu \mathrm{m}$ \\
\hline \hline
\end{tabular}

Table 5 - Lay-up and properties of the laminate containingK13D2U 
Fermi National Accelerator Laboratory

Giobatta Lanfranco Silicon Engineering Group - Mechanical Dep.

\begin{tabular}{|c|c|c||}
\hline \hline Material & $\begin{array}{c}\text { CTE } \\
{\left[\mu \mathrm{m} / \mathrm{m}-{ }^{\circ} \mathrm{C}\right]}\end{array}$ & $\begin{array}{c}\text { Young's modulus } \\
{[\mathrm{Gpa}(\mathrm{Msi})]}\end{array}$ \\
\hline Silicon & 2.5 & $112.4(16.3)$ \\
Kapton & 20 & $2.8(0.406)$ \\
Epoxy & 43.8 & $3.2(0.464)$ \\
Ceramic (MACOR) & 7.4 & $66.9(9.7)$ \\
Polyetheretherketone (PEEK) & 40 & $3.8(0.551)$ \\
Beryllia (BeO) & 8.4 & $311(45.1)$ \\
Rohacell 51 A & 33 & $0.07(0.01)$ \\
\hline
\end{tabular}

Table 6-CTE and elastic modulus of materials used in the stave

In the following pictures (Figure 2 - Figure 13) it is possible to see the behaviour that the structure exhibits with a $30 \mathrm{~K}$ temperature drop. Rohacell reduces the membrane behaviour of the silicon sensor and together with a third couple of supports half the span helps to greatly reduce the sag; the K13D2U heat spreader in conjunction with the $\mathrm{K} 139\left[90^{\circ}-0^{\circ}\right]$ restrain the silicon from elongating longitudinally. The stresses in the sensor never rise to a dangerous level, the safety factor always being at least five ${ }^{5}$. Table 7 summarizes the maximum and minimum values of vertical and longitudinal displacement and the longitudinal stress.

\footnotetext{
${ }^{5}$ The silicon compressive yield strength is about $120 \mathrm{MPa}$.
} 
Fermi National Accelerator Laboratory

Giobatta Lanfranco Silicon Engineering Group - Mechanical Dep.

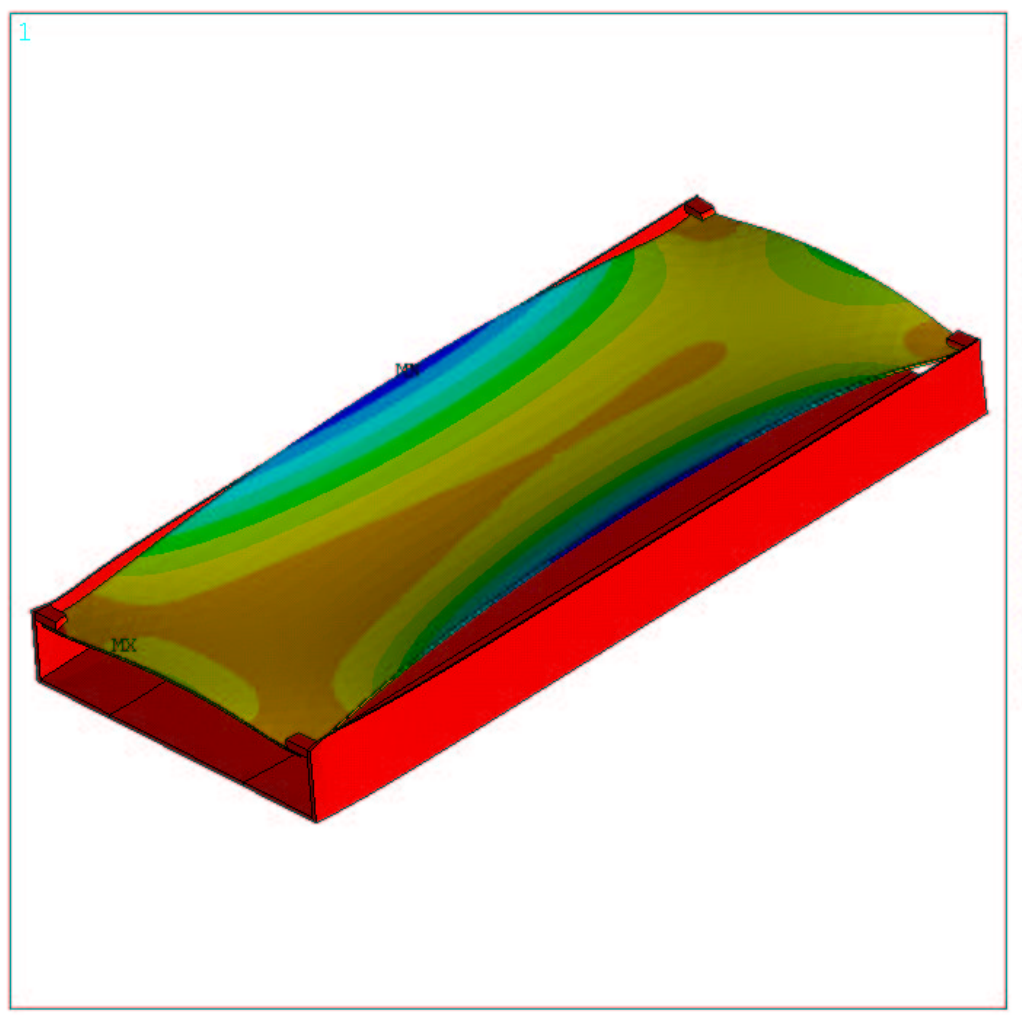

$$
\begin{aligned}
& \text { ANSYS } 5.5 .3 \\
& \text { JUN } 52001 \\
& 12: 13: 28 \\
& \text { NODAL SOLUTION } \\
& \text { STEP }=1 \\
& \text { SUB }=1 \\
& \text { T IME }=1 \\
& \text { UY } \\
& \text { RSYS }=0
\end{aligned}
$$

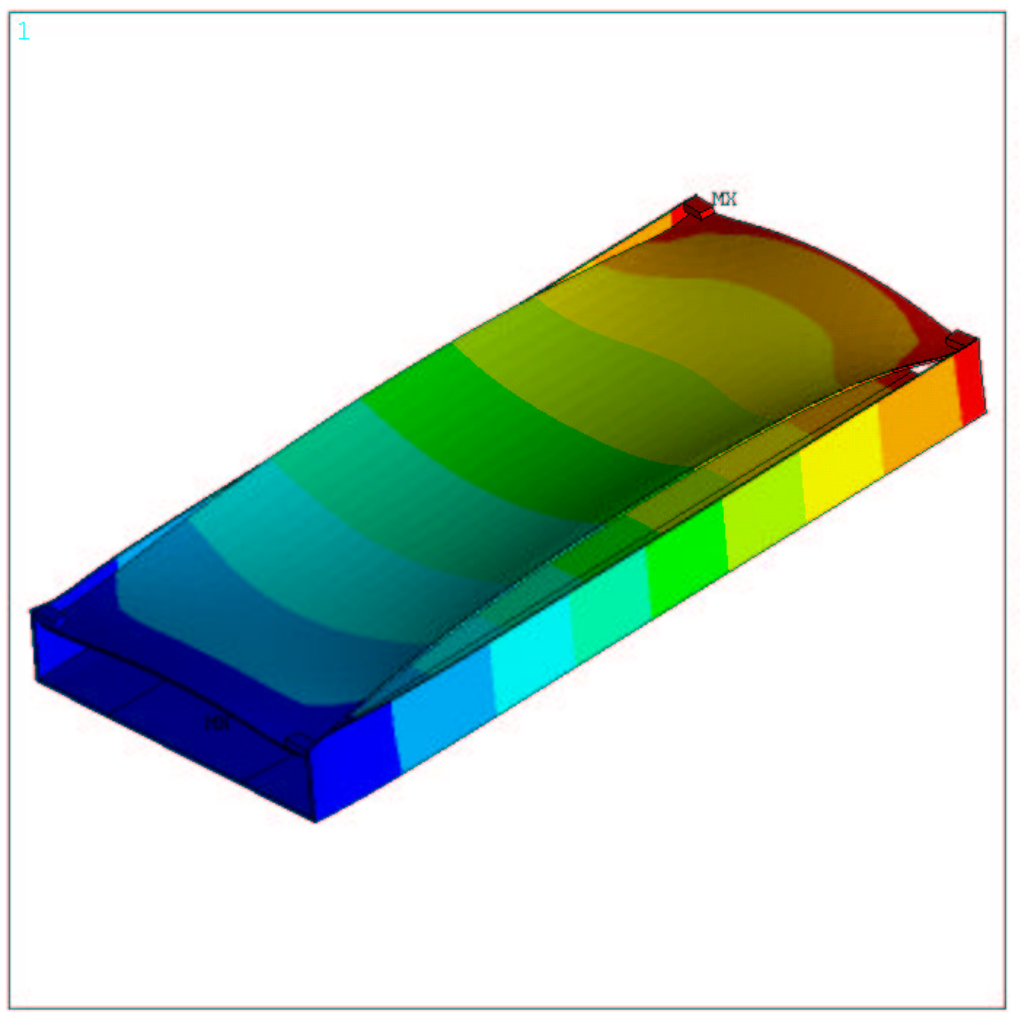

ANSYS $5 \cdot 5 \cdot 3$

JUN $5 \quad 2001$

$12: 16: 06$

NODAL SOLUTION

$\mathrm{STEP}=1$

SUB $=1$

TIME $=1$

U2

(AVG)

RSYS $=0$

PowerGraphics

EFACET $=1$

AVRES $=$ Mat

$\mathrm{DMX}=.011036$

$\mathrm{SMN}=-.134 \mathrm{E}-03$

SMX $=.010118$

$-.134 \mathrm{E}-03$

.001005

.001005
.002145

.003284

.004423

.005562

.006701

.00784

.008979

.010118

Figure 2 - From top to bottom: vertical and longitudinal displacements [mm] in half stave structure $100 \mathrm{~mm}$ long [Shell: K139 + Boron Prepreg; heat spreader perfectly matching silicon CTE] 
Fermi National Accelerator Laboratory

Giobatta Lanfranco Silicon Engineering Group - Mechanical Dep.

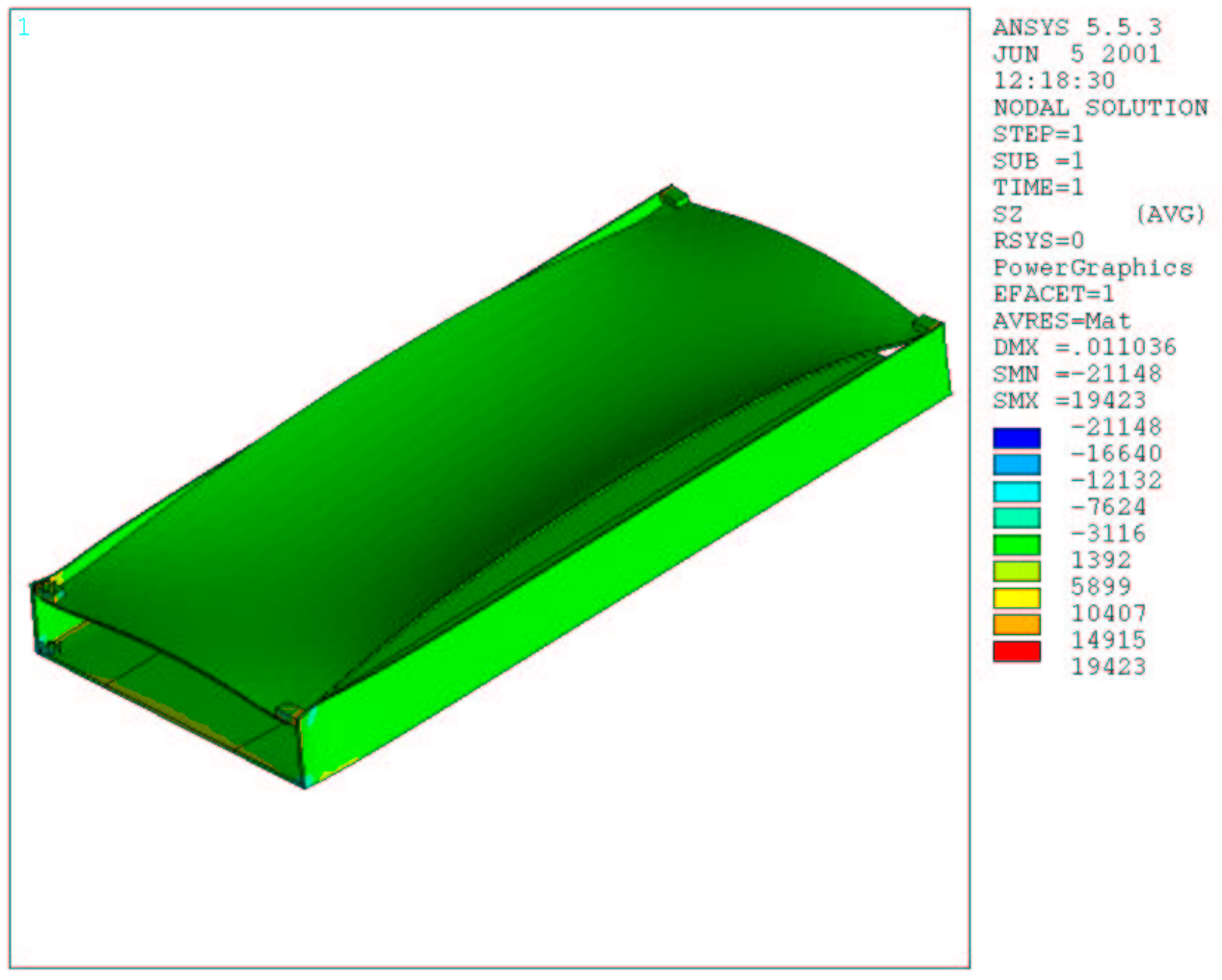

Figure 3 - Longitudinal stresses [ $\mathrm{kPa}, \sim 1 / 7$ psi] in half stave structure $100 \mathrm{~mm}$ long [Shell: K139 + Boron Prepreg; heat spreader perfectly matching silicon CTE] 
Fermi National Accelerator Laboratory

Giobatta Lanfranco Silicon Engineering Group - Mechanical Dep.

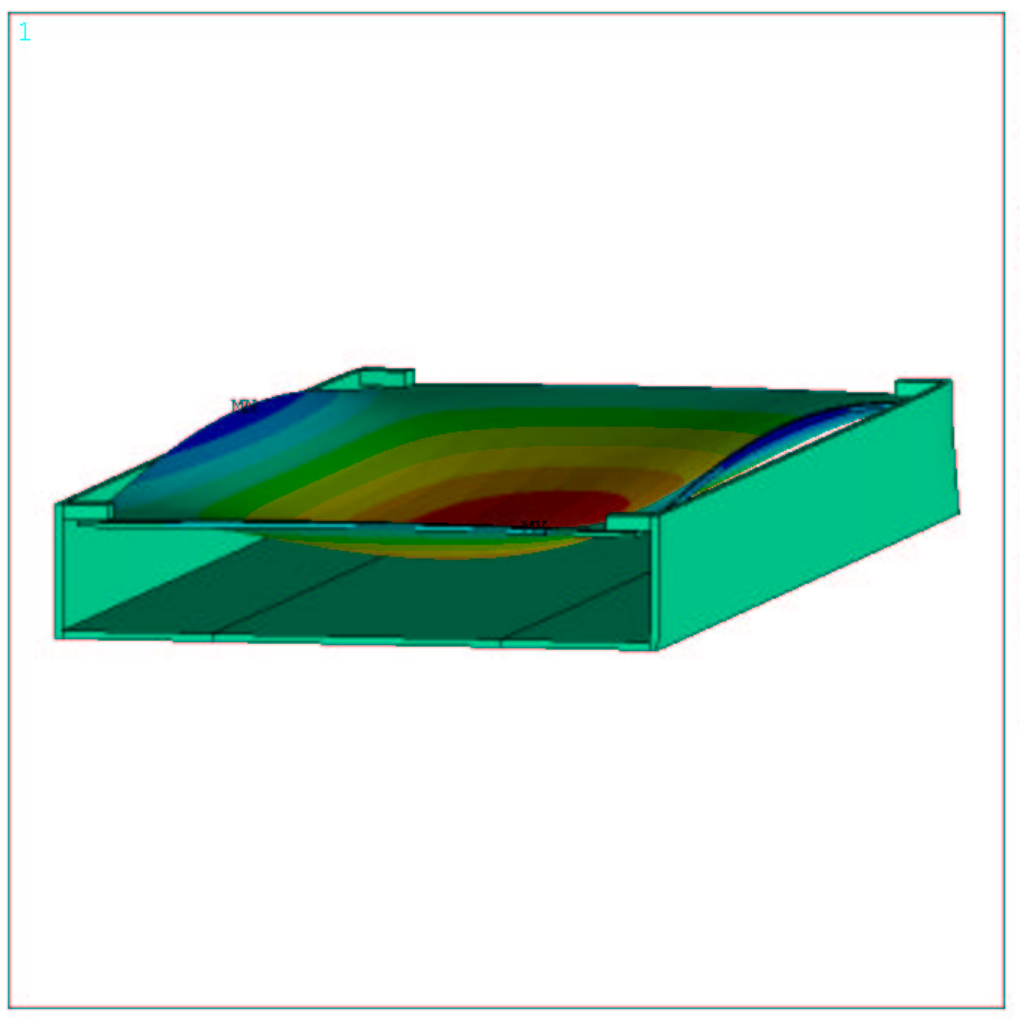

ANSYS 5.5 .3

JUN 52001

$14: 25: 54$

NODAL SOLUTION

STEP $=1$

SUB $=1$

TIME $=1$

UY

RSYS $=0$

PowerGraphics

EFACET $=1$

AVRE $S=$ Mat

DMX $=.207779$

SMN $=-.109075$

$\operatorname{SMX}=.20726$

$-.109075$

$-.073927$

$-.038779$

$-.00363$

.031518

.066667

.101815

.136963

.172112

.20726

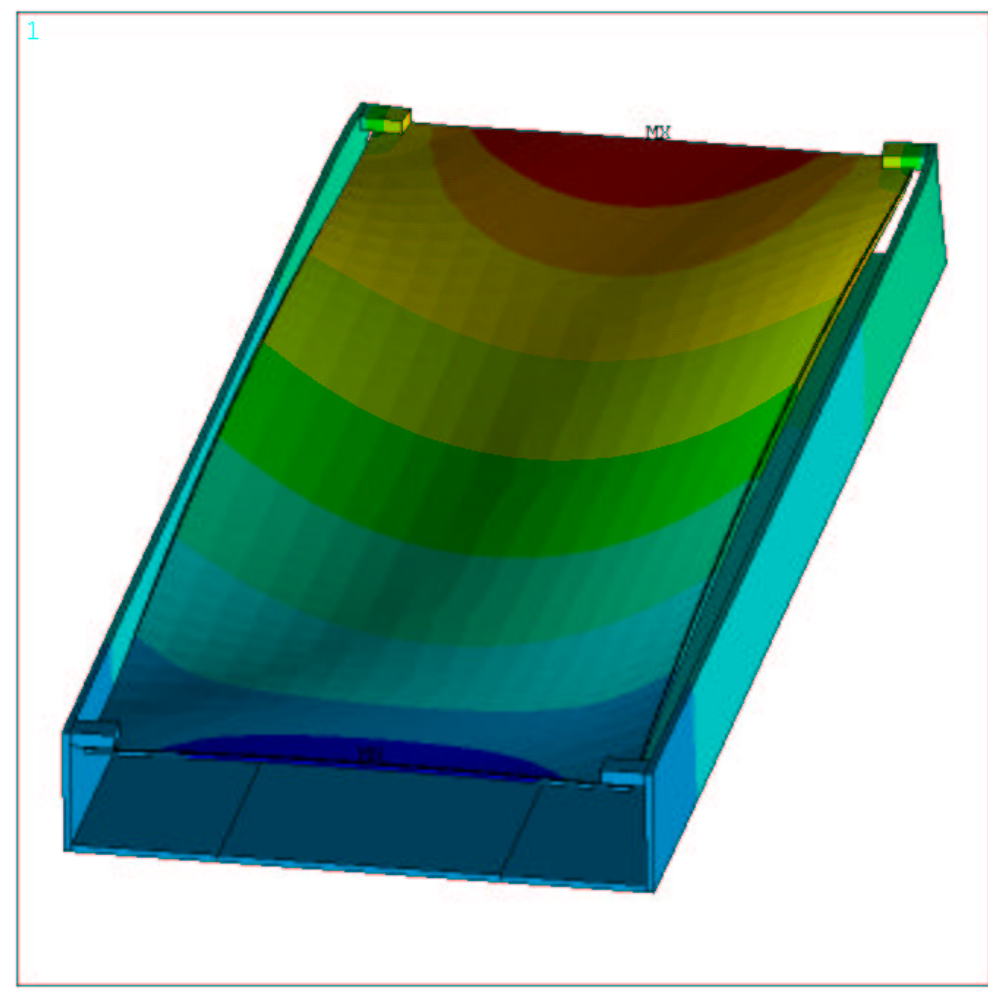

ANSYS 5.5 .3

JUN 52001

$14: 27: 05$

NODAL SOLUTION

$\mathrm{STEP}=1$

SUB $=1$

TIME $=1$

UZ

(AVG)

RSYS $=0$

PowerGraphics

EFACET $=1$

AVRE $S=$ Mat

DMX $=.207779$

SMN $=-.010302$

$\mathrm{SMX}=.041565$

SMX $=.041565$

.010302
-.004539

.001224

.006987

.01275

.018513

.024276

.030039

.035802

.041565

Figure 4 - From top to bottom: vertical and longitudinal displacements [mm] in half stave structure $100 \mathrm{~mm}$ long [Shell: K139 + Boron Prepreg; KAPTON heat spreader 
Fermi National Accelerator Laboratory

Giobatta Lanfranco Silicon Engineering Group - Mechanical Dep.

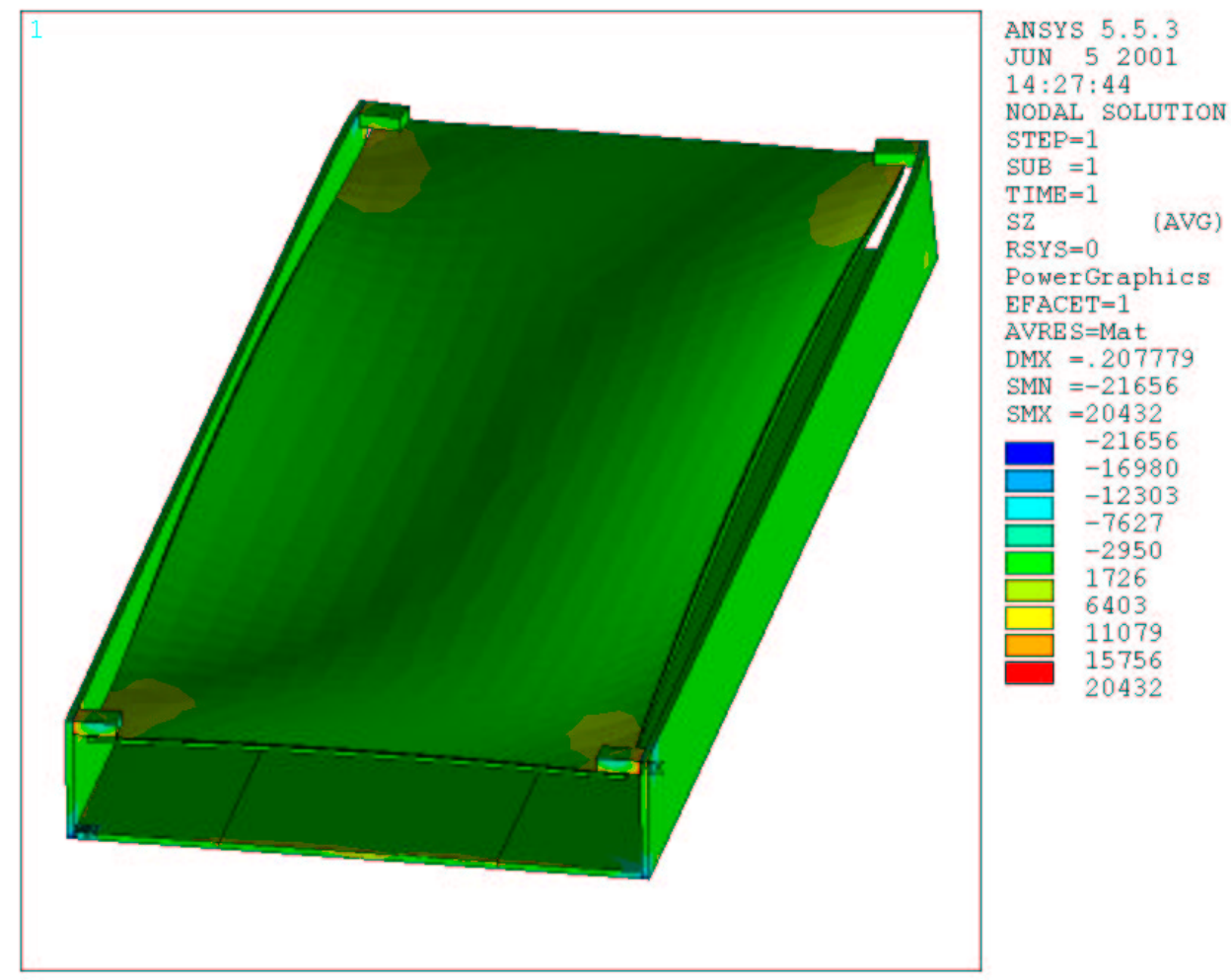

Figure 5 - Longitudinal stresses [kPa, 1/7 psi] in half stave structure $100 \mathrm{~mm}$ long [Shell: K139 + Boron Prepreg; KAPTON heat spreader] 
Fermi National Accelerator Laboratory

Giobatta Lanfranco Silicon Engineering Group - Mechanical Dep.
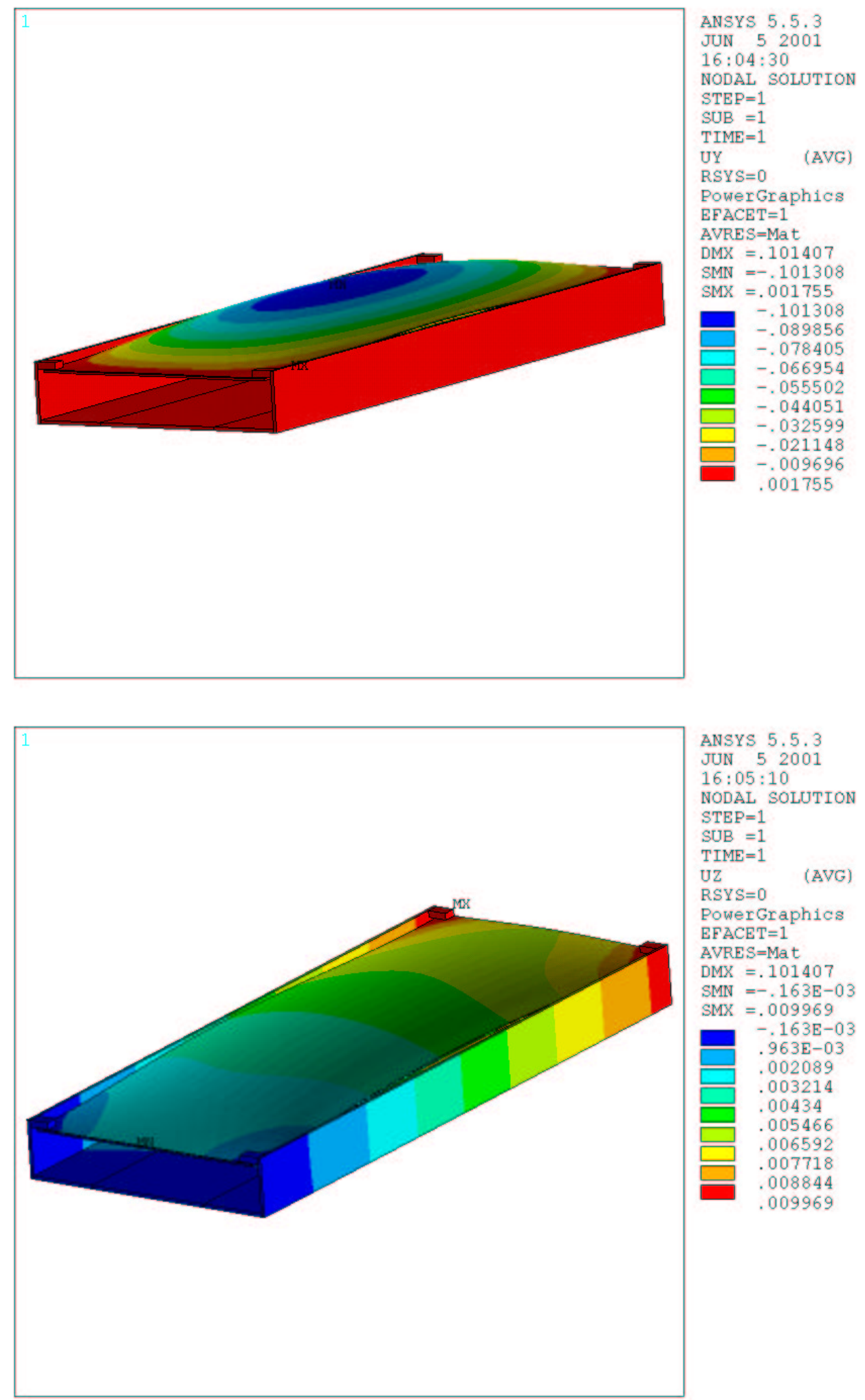

Figure 6 - From top to bottom: vertical and longitudinal displacements [mm] in half stave structure $100 \mathrm{~mm}$ long [Shell: K139 + Boron Prepreg; K13D2U heat spreader 
Fermi National Accelerator Laboratory

Giobatta Lanfranco Silicon Engineering Group - Mechanical Dep.

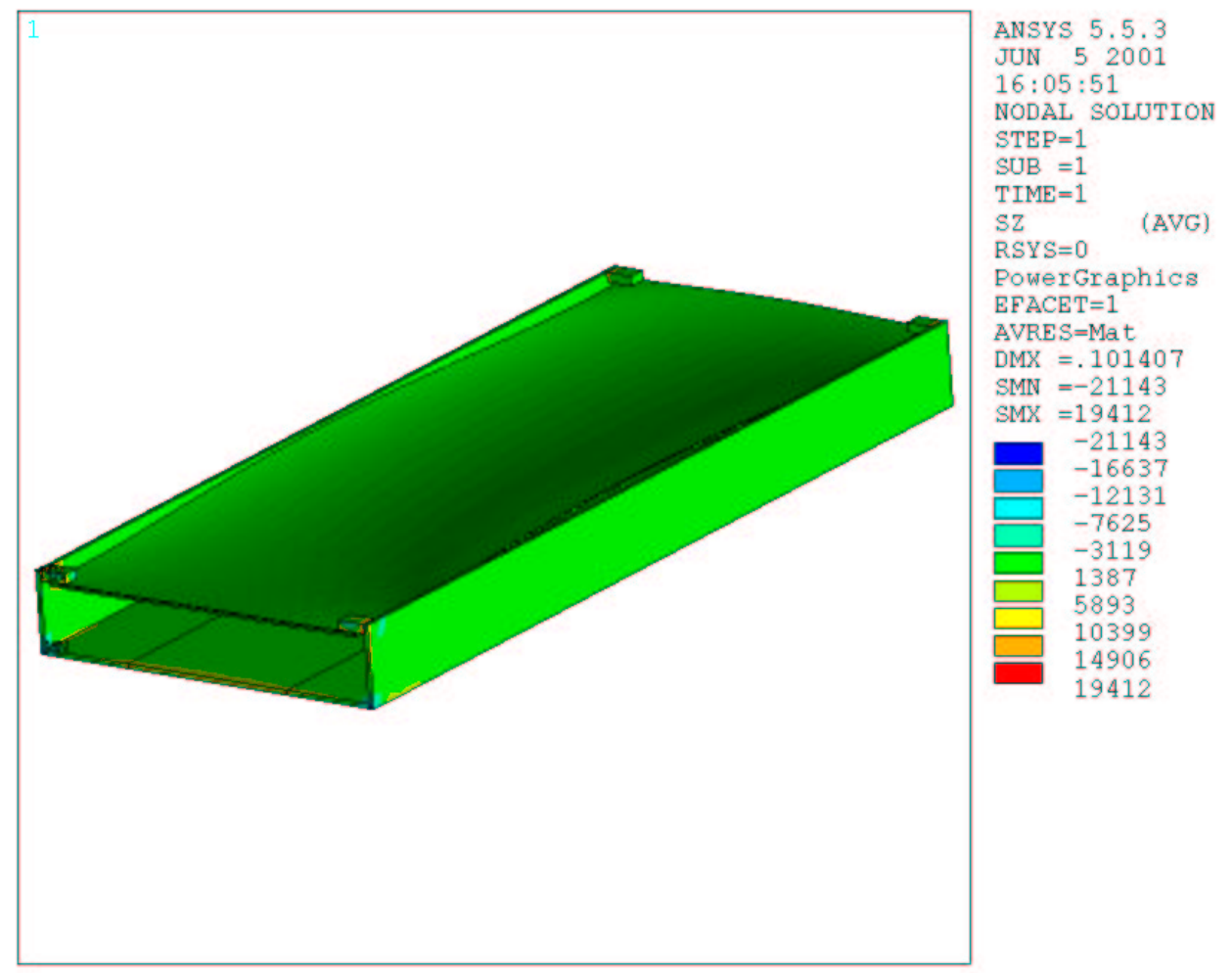

Figure 7 - Longitudinal stresses [kPa, 1/7 psi] in half stave structure $100 \mathrm{~mm}$ long [Shell: K139 + Boron Prepreg; K13D2U heat spreader] 
Fermi National Accelerator Laboratory

Giobatta Lanfranco Silicon Engineering Group - Mechanical Dep.
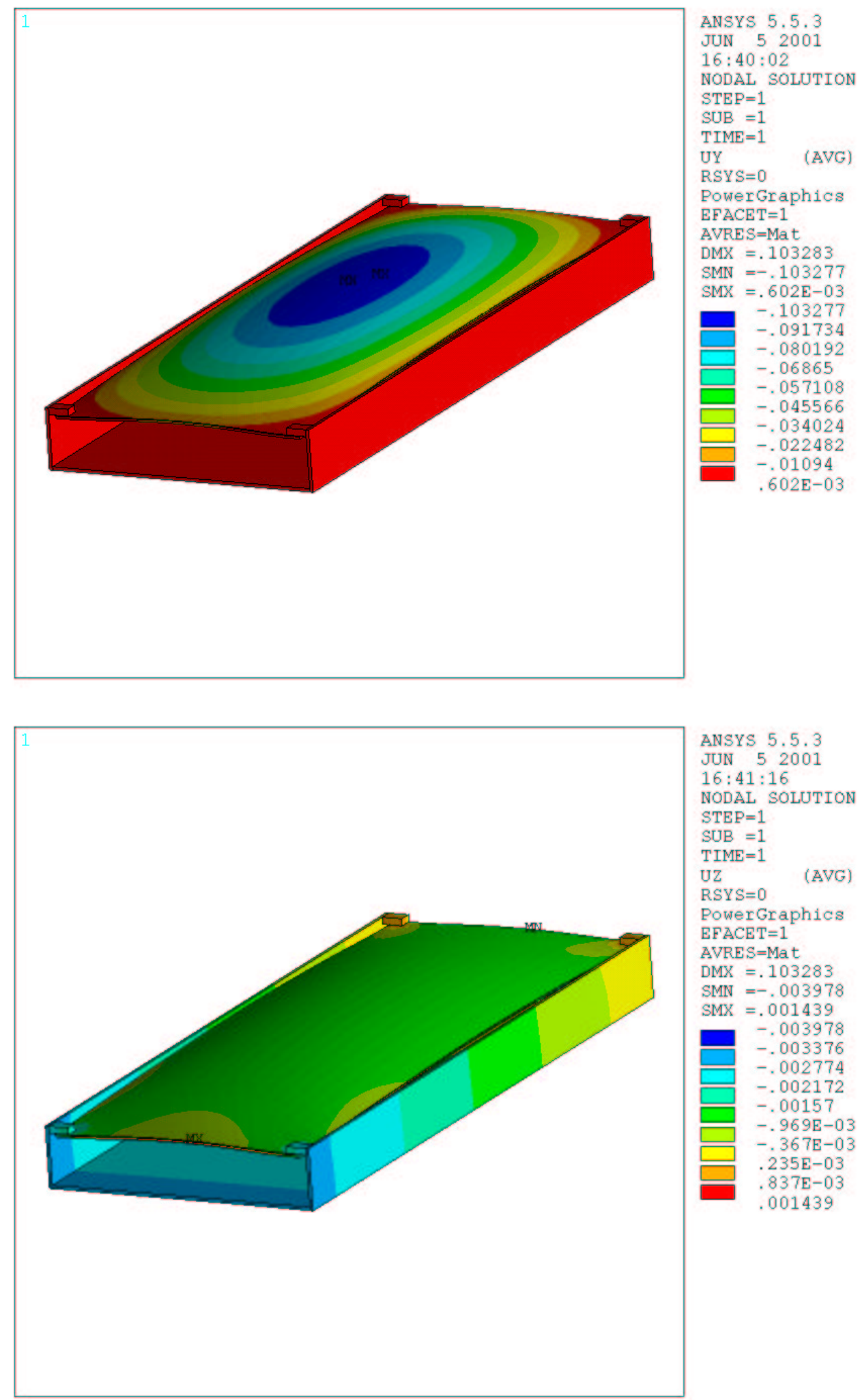

Figure 8 - From top to bottom: vertical and longitudinal displacements [mm] in half stave structure $100 \mathrm{~mm}$ long [Shell: K139 $\left(90^{\circ}-0^{\circ}-90^{\circ}-0^{\circ}-90^{\circ}-0^{\circ}-90^{\circ}\right)$; K13D2U heat spreader 
Fermi National Accelerator Laboratory

Giobatta Lanfranco Silicon Engineering Group - Mechanical Dep.

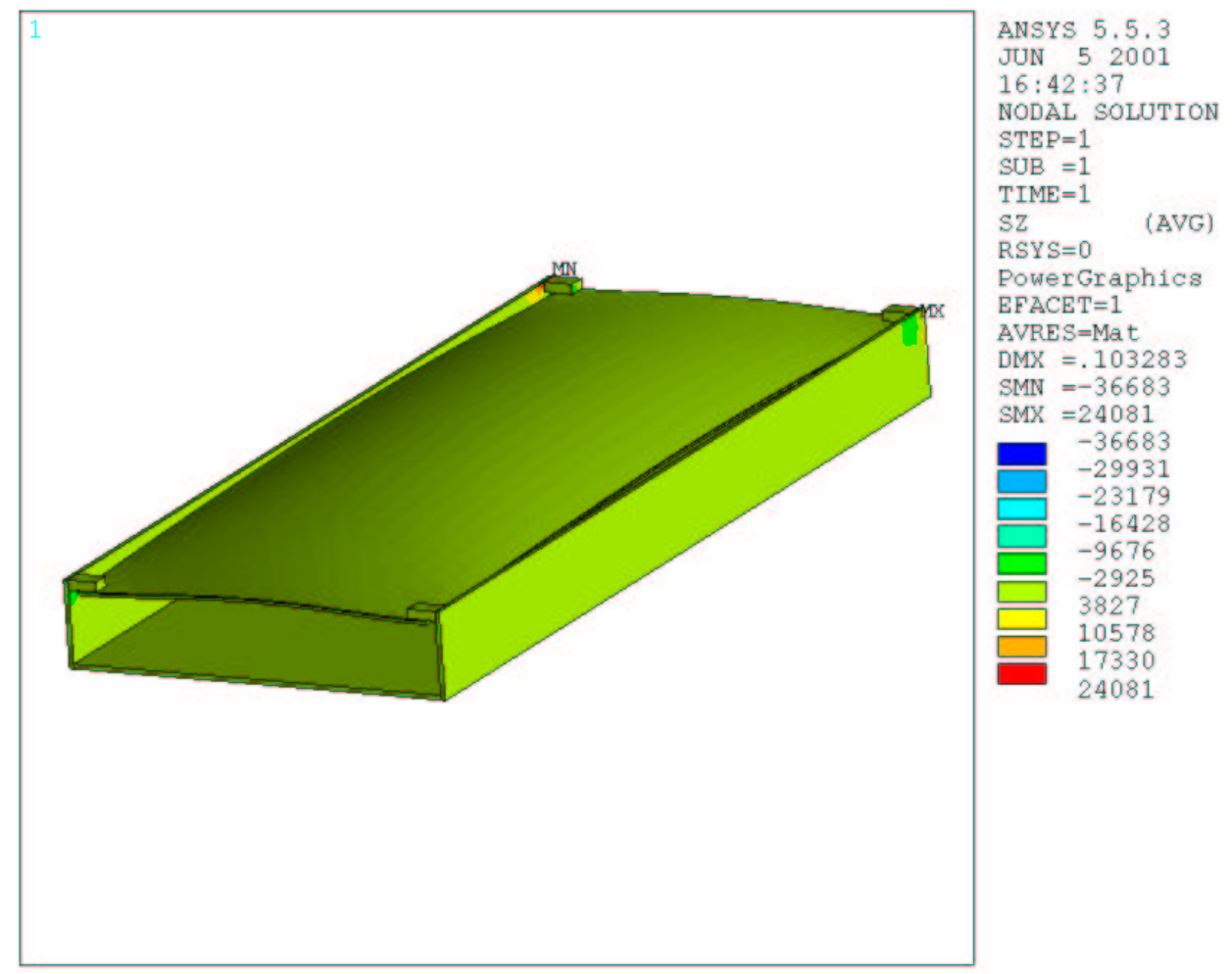

Figure 9 - Longitudinal stresses $\left[\mathrm{kPa}, \sim 1 / 7 \mathrm{psi}\right.$ ] in half stave structure $100 \mathrm{~mm}$ long [Shell: K139 $\left(90^{\circ}-0^{\circ}-90^{\circ}-0^{\circ}-90^{\circ}-0^{\circ}\right.$ $\left.90^{\circ}\right)$; K13D2U heat spreader] 
Fermi National Accelerator Laboratory

Giobatta Lanfranco Silicon Engineering Group - Mechanical Dep.

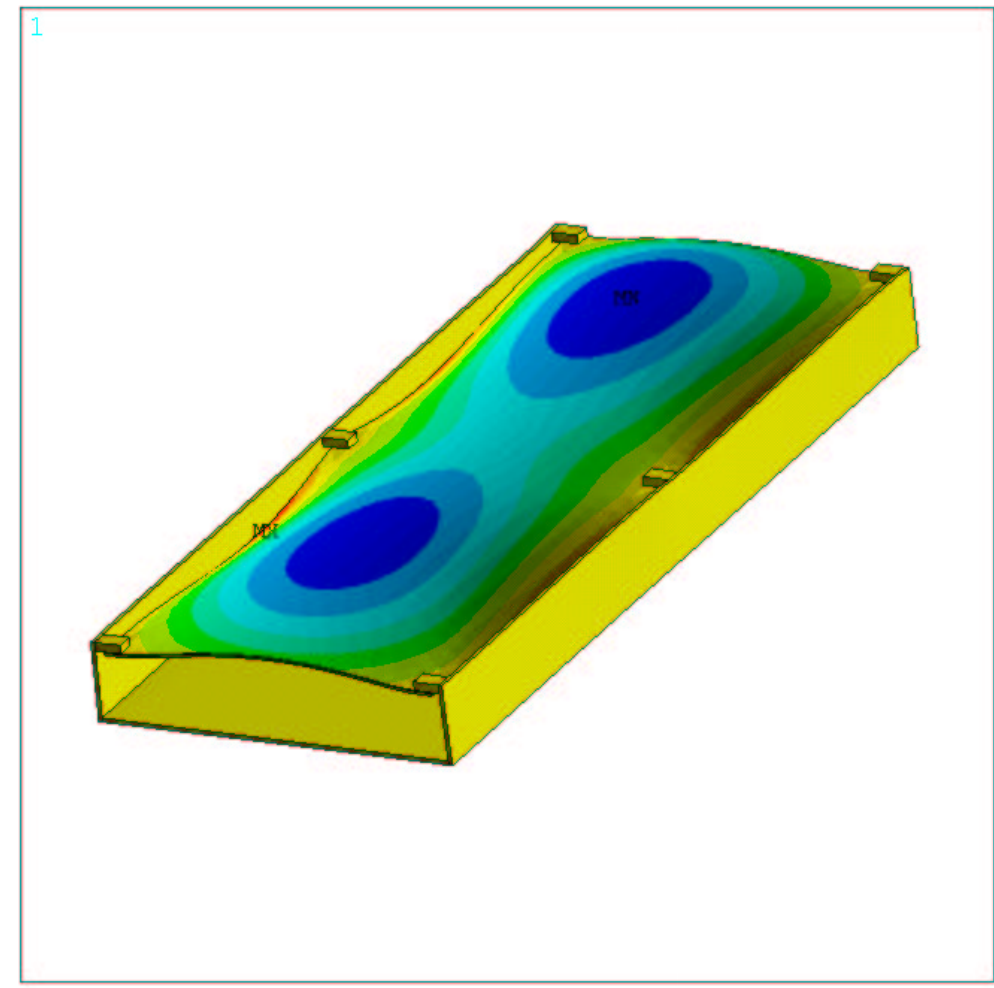

ANSYS $5 \cdot 5 \cdot 3$

JUN 52001

$17: 17: 51$

NODAL SOLUTION

$\mathrm{STEP}=1$

SUB $=1$

TIME $=1$

UY

(AVG)

RSYS $=0$

PowerGraphics

EFACET $=1$

AVRE $S=$ Mat

DMX $=.044666$

SMN $=-.044639$

SMX $=.018114$

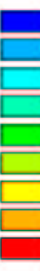

$-.044639$

$-.037667$

$-.030694$

$-.023721$

$-.016749$

$-.016$

$-.009776$

$-.002804$

.004169

.011141

.018114

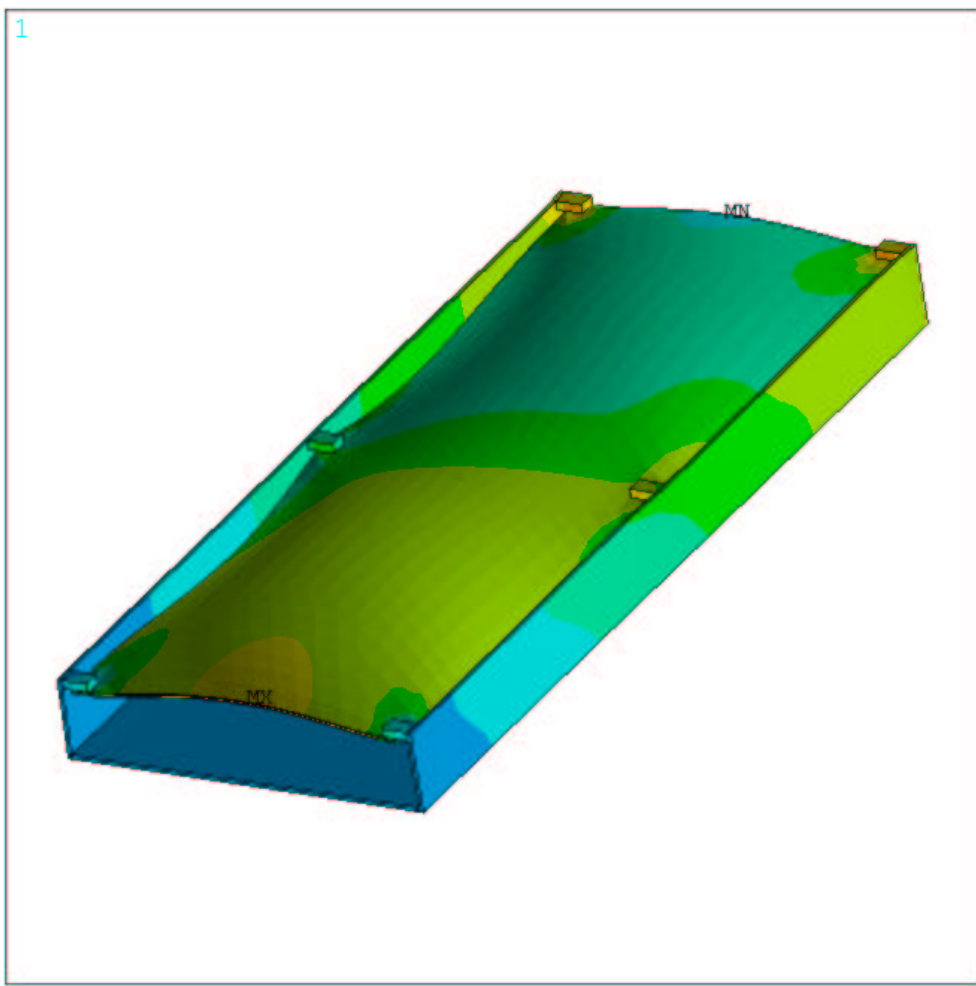

ANSYS 5.5 .3

JUN 52001

$17: 34: 47$

NODAL SOLUTION

STEP $=1$

SUB $=1$

TIME $=1$

UZ

RSYS $=0$

PowerGraphics

EFACET $=1$

AVRE $S=$ Mat

DMX $=.044666$

SMN $=-.003657$

SMX $=.001808$

$-.003657$

$-.00305$

$-.002442$

-.002442
-.001835

$-.001228$

$-.621 \mathrm{E}-03$

$-.138 \mathrm{E}-04$

$.593 \mathrm{E}-03$

.001201

.001808

Figure 10 - From top to bottom: vertical and longitudinal displacements [mm] in half stave structure $100 \mathrm{~mm}$ long [Shell: $\mathrm{K} 139\left(90^{\circ}-0^{\circ}-90^{\circ}-0^{\circ}-90^{\circ}-0^{\circ}-90^{\circ}\right)$; K13D2U heat spreader; 6 supports] 
Fermi National Accelerator Laboratory

Giobatta Lanfranco Silicon Engineering Group - Mechanical Dep.

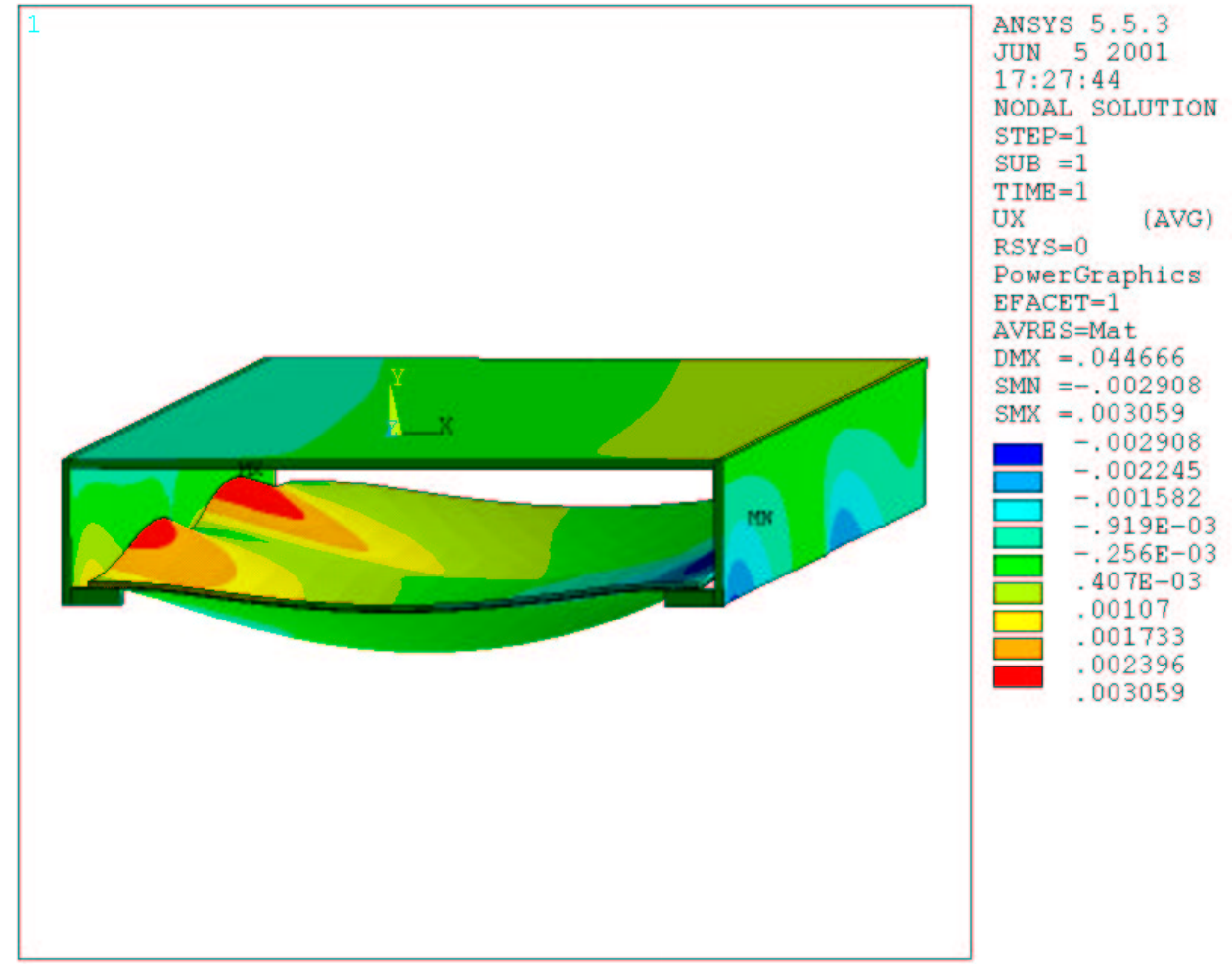

Figure 11 - Lateral displacements [mm] in half stave structure $100 \mathrm{~mm}$ long [Shell: K139 $\left(90^{\circ}-0^{\circ}-90^{\circ}-0^{\circ}-90^{\circ}-0^{\circ}-90^{\circ}\right)$; K13D2U heat spreader; 6 supports] 
Fermi National Accelerator Laboratory

Giobatta Lanfranco Silicon Engineering Group - Mechanical Dep.

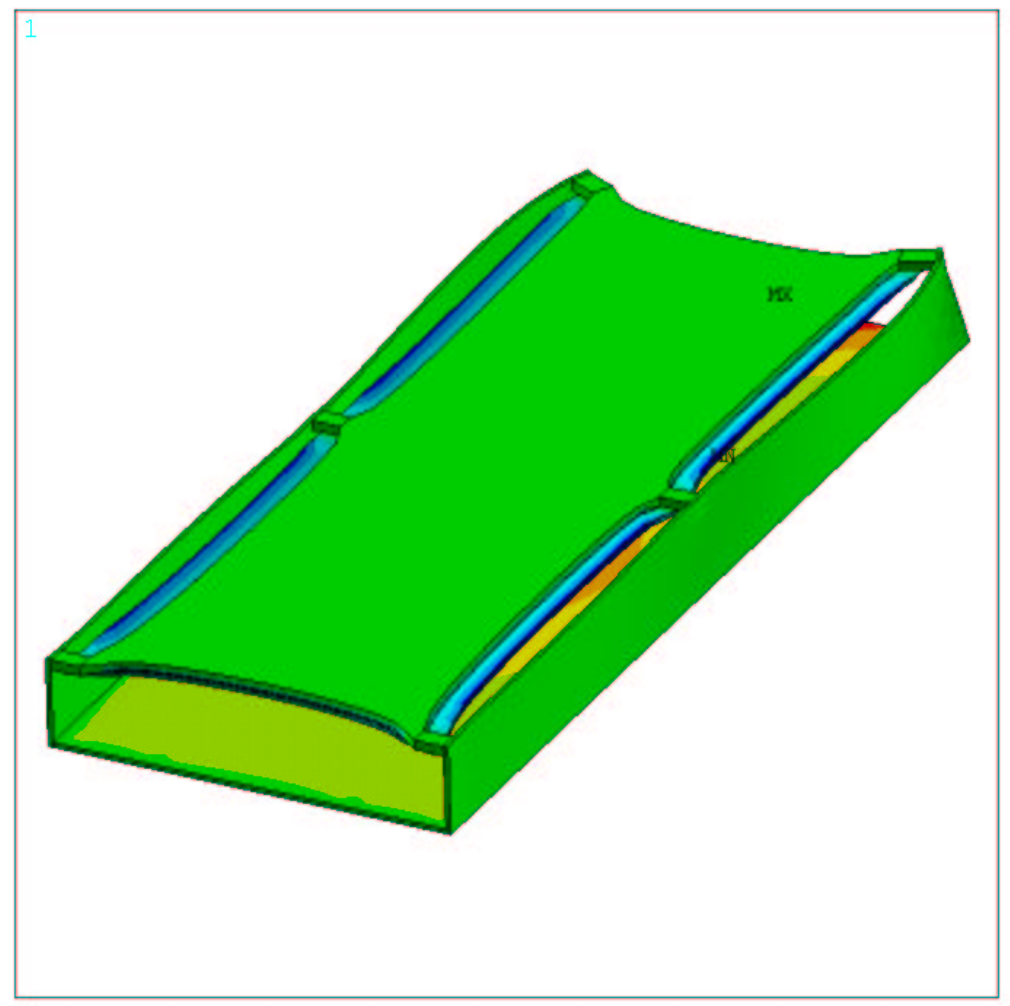

ANSYS 5.5 .3

JUN 62001

$10: 38: 09$

NODAL SOLUTION

$\mathrm{STEP}=1$

SUB $=1$

TIME $=1$

UY

RSYS $=0$

PowerGraphics

EFACET $=1$

AVRE $S=$ Mat

DMX $=.034413$

$\mathrm{SMN}=-.007038$

SMX $=.006194$

$\begin{aligned} \mathrm{SMX}= & .006194 \\ & -.007038\end{aligned}$

$-.005568$

$-.004098$

$-.002627$

$-.001157$

$\square .313 \mathrm{E}-03$

$\square .003254$

.004724

.006194

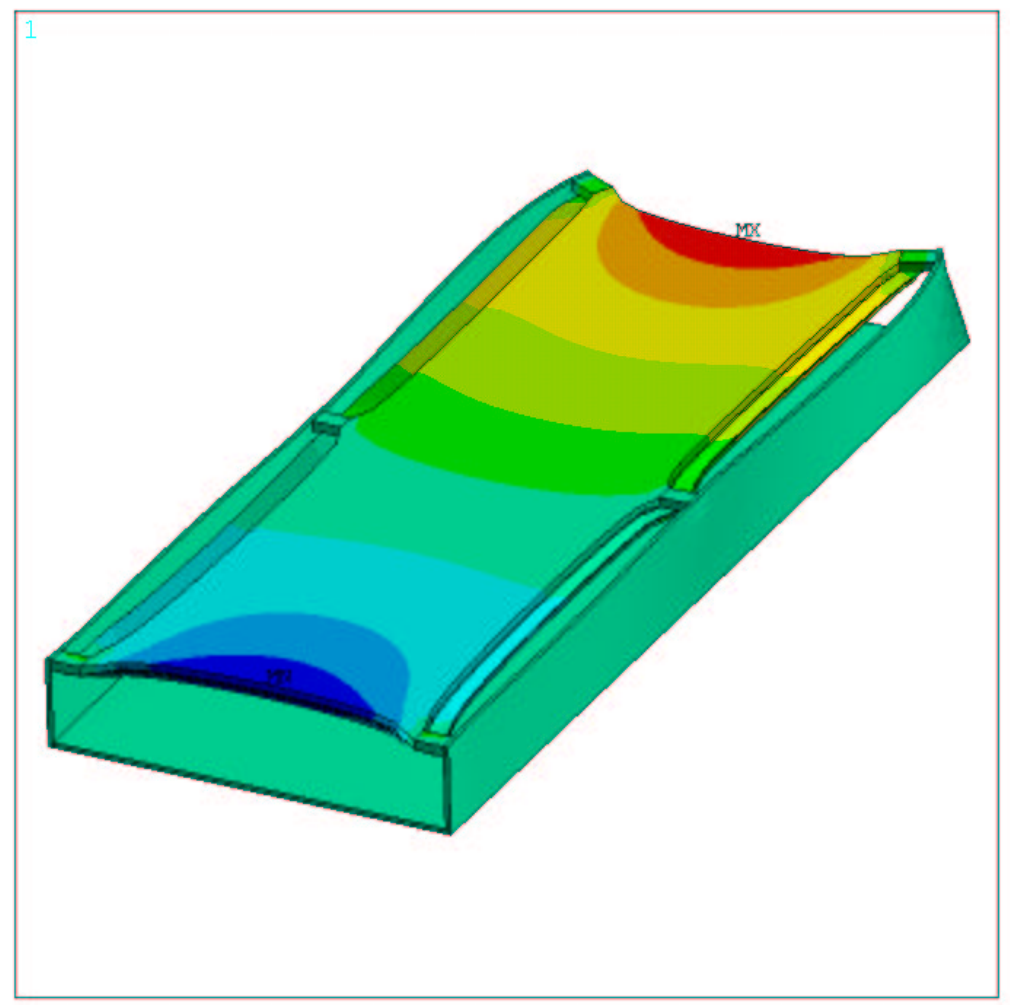

ANSYS 5.5 .3

JUN 62001

$10: 39: 02$

NODAL SOLUTION

$\mathrm{STEP}=1$

$\mathrm{SUB}=1$

TIME $=1$

$\mathrm{UZ}$

(AVG)

RSYS $=0$

PowerGraphics

EFACET $=1$

AVRE S=Mat

$\mathrm{DMX}=.034413$

$\mathrm{SMN}=-.026807$

SMX $=.034413$

$-.026807$

$-.020004$

$-\quad-.013202$

$-.0064$

$.402 \mathrm{E}-0$

. .007204

.014006

.020809

.027611
.034413

Figure 12 - From top to bottom: vertical and longitudinal displacement [mm] in half stave structure $100 \mathrm{~mm}$ long [Shell: $\mathrm{K} 139\left(90^{\circ}-0^{\circ}-90^{\circ}-0^{\circ}-90^{\circ}-0^{\circ}-90^{\circ}\right)$; K13D2U heat spreader; Rohacell core; 6 supports] 
Fermi National Accelerator Laboratory

Giobatta Lanfranco Silicon Engineering Group - Mechanical Dep.
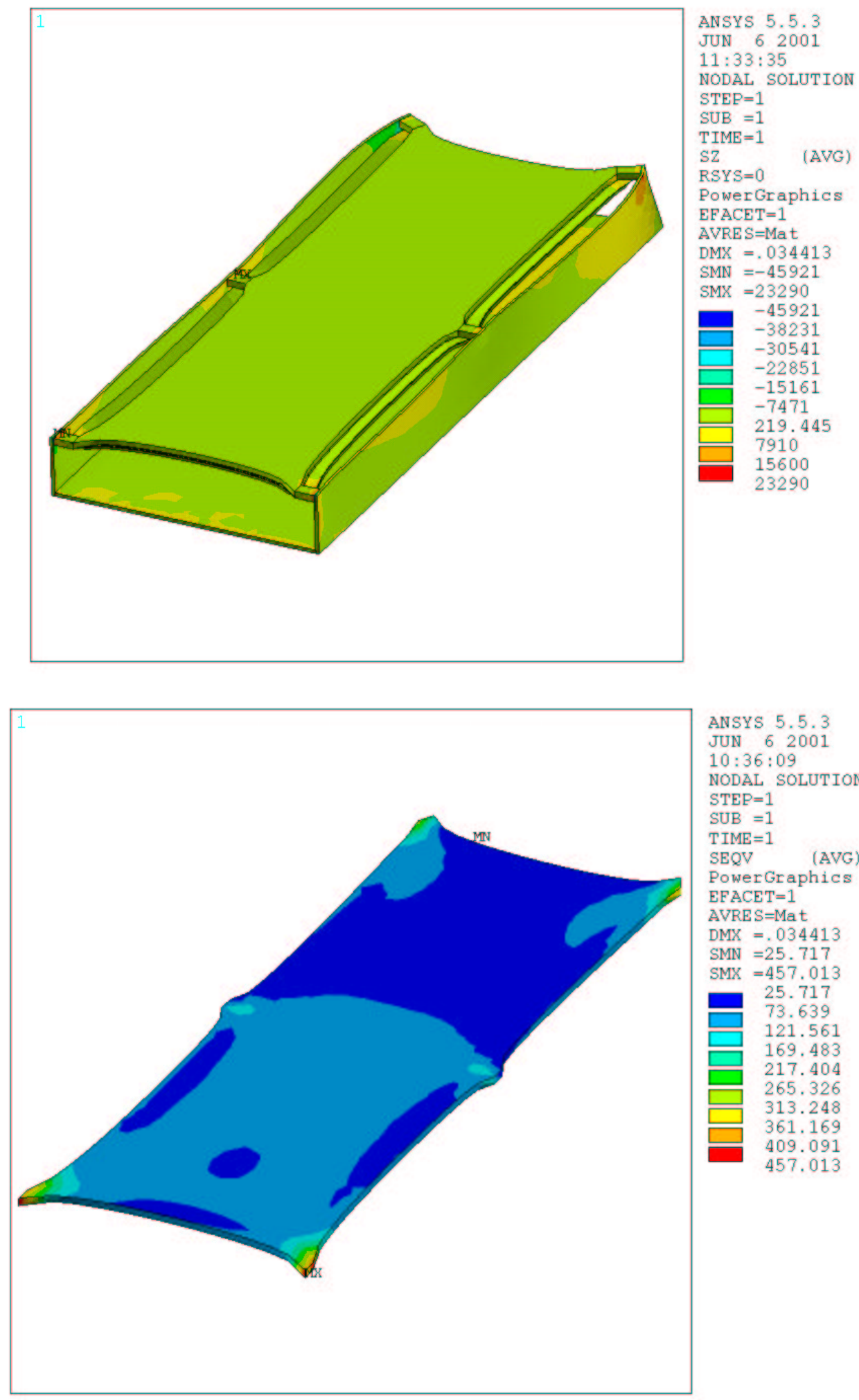

ANSYS 5.5 .3

JUN 62001

$10: 36: 09$

NODAL SOLUTION

STEP $=1$

$\mathrm{SUB}=1$

TIME $=1$

SEQV

(AVG)

PowerGraphics

EFACET $=1$

AVRES $=$ Mat

$\mathrm{DMX}=.034413$

$\mathrm{SMN}=25.717$

SMX $=457.013$

$\begin{array}{r}25.717 \\ \hline\end{array}$

73.639

121.561

217.404

$\square \quad 265.326$

313.248

? 361.169

409.091
457.013

Figure 13 - From top to bottom: longitudinal stresses in half structure and Von Mises stress distribution in Rohacell [kPa, $1 / 7 \mathrm{psi}$ - Rohacell strength $1600 \mathrm{kPa}(230 \mathrm{psi})$ [Shell: K139 $\left(90^{\circ}-0^{\circ}-90^{\circ}-0^{\circ}-90^{\circ}-0^{\circ}-90^{\circ}\right)$; K13D2U heat spreader; Rohacell core; 6 supports] 
Fermi National Accelerator Laboratory

Giobatta Lanfranco Silicon Engineering Group - Mechanical Dep.

\begin{tabular}{|c|c|c|c|c|c|c|c|c|}
\hline Case & $\min U Y$ & $\max U Y$ & $\min U Z$ & $\max U Z$ & & ess & ma & ess \\
\hline \multirow[b]{2}{*}{1} & $\mu \mathrm{m}$ & $\mu \mathrm{m}$ & $\mu \mathrm{m}$ & $\mu \mathrm{m}$ & $\mathrm{kPa}$ & psi & $\mathrm{kPa}$ & psi \\
\hline & -9 & 0 & 0 & 10 & -21148 & -3066 & 19423 & 2816 \\
\hline 2 & -109 & 207 & -10 & 41 & -21656 & -3140 & 20432 & 2963 \\
\hline 3 & -101 & 2 & 0 & 10 & -21143 & -3066 & 19412 & 2815 \\
\hline 4 & -103 & 0 & -4 & 1 & -36683 & -5319 & 24081 & 3492 \\
\hline 5 & -44 & 18 & -4 & 2 & -43258 & -6272 & 22989 & 3333 \\
\hline 6 & -7 & 6 & -27 & 34 & -45921 & -6659 & 23290 & 3377 \\
\hline \multicolumn{9}{|l|}{ Case } \\
\hline 1 & \multicolumn{8}{|c|}{ Shell: K139 + Boron Prepreg; heat spreader perfectly matching silicon CTE } \\
\hline 2 & \multicolumn{8}{|c|}{ Shell: K139 + Boron Prepreg; KAPTON heat spreader } \\
\hline 3 & \multicolumn{8}{|c|}{ Shell: K139 + Boron Prepreg; K13D2U heat spreader } \\
\hline 4 & \multicolumn{8}{|c|}{ Shell: K139 $\left(90^{\circ}-0^{\circ}-\ldots\right)$; K13D2U heat spreader } \\
\hline 5 & \multicolumn{8}{|c|}{ Shell: K139 $\left(90^{\circ}-0^{\circ}-\ldots\right)$; K13D2U heat spreader; 6 supports } \\
\hline 6 & \multicolumn{8}{|c|}{ Shell: K139 (90 $\left.90^{\circ}-0^{\circ}-90^{\circ}-\ldots\right)$; K13D2U heat spreader; Rohacell core; 6 supports } \\
\hline
\end{tabular}

Table 7 - Summarize on thermal mismatch finite element analysis

\section{A more realistic case}

The study at the previous paragraph allows identifying the better performing materials in order to produce a more realistic model (Figure 14). The model represents a chain of three sensors per side. The hybrids are located, as already mentioned, according to two different configurations: alternate (configuration \#1) and facing (configuration \#2). The heat load has been spread on two areas $10 \times 37.2$ $\mathrm{mm}^{2}$ per hybrid, $1 \mathrm{~mm}$ off the hybrid edge, each area having $2.5 \mathrm{~W}$ heat load. The coolant bulk temperature is assumed equal to $-10{ }^{\circ} \mathrm{C}$ with a film coefficient of $2000 \mathrm{~W} / \mathrm{mK}$.

In Figure 14 the modeled materials are listed and it is shown a front and isometric view of the modeled stave.

It can be seen that the temperature profile follows a similar pattern for both configurations, with a slightly lower maximum temperature for configuration 1 because of the lower heat density in the structure. Applying the temperature distribution derived from the chips heat generation, because of the lower off-set from symmetry, configuration 2 exhibits a lower vertical displacements with comparable longitudinal contraction and thermal induced stresses. 
Fermi National Accelerator Laboratory

Giobatta Lanfranco Silicon Engineering Group - Mechanical Dep.

What aforementioned, can be seen in Figure 15 - Figure 20 and it is summarized in Table 8.

\begin{tabular}{||c|c||}
\hline \multicolumn{1}{|c|}{ Component } & Material \\
\hline Ladder core & Rohacell \\
Heat spreader & K13D2U \\
Sensor & silicon \\
Outer shell & K139 $\left[90^{\circ}-0^{\circ}\right]$ \\
Supporting blocks & MACOR \\
Glue & Epoxy \\
Tubing & PEEK \\
Substrate & BeO \\
HDI & Kapton \\
\hline
\end{tabular}
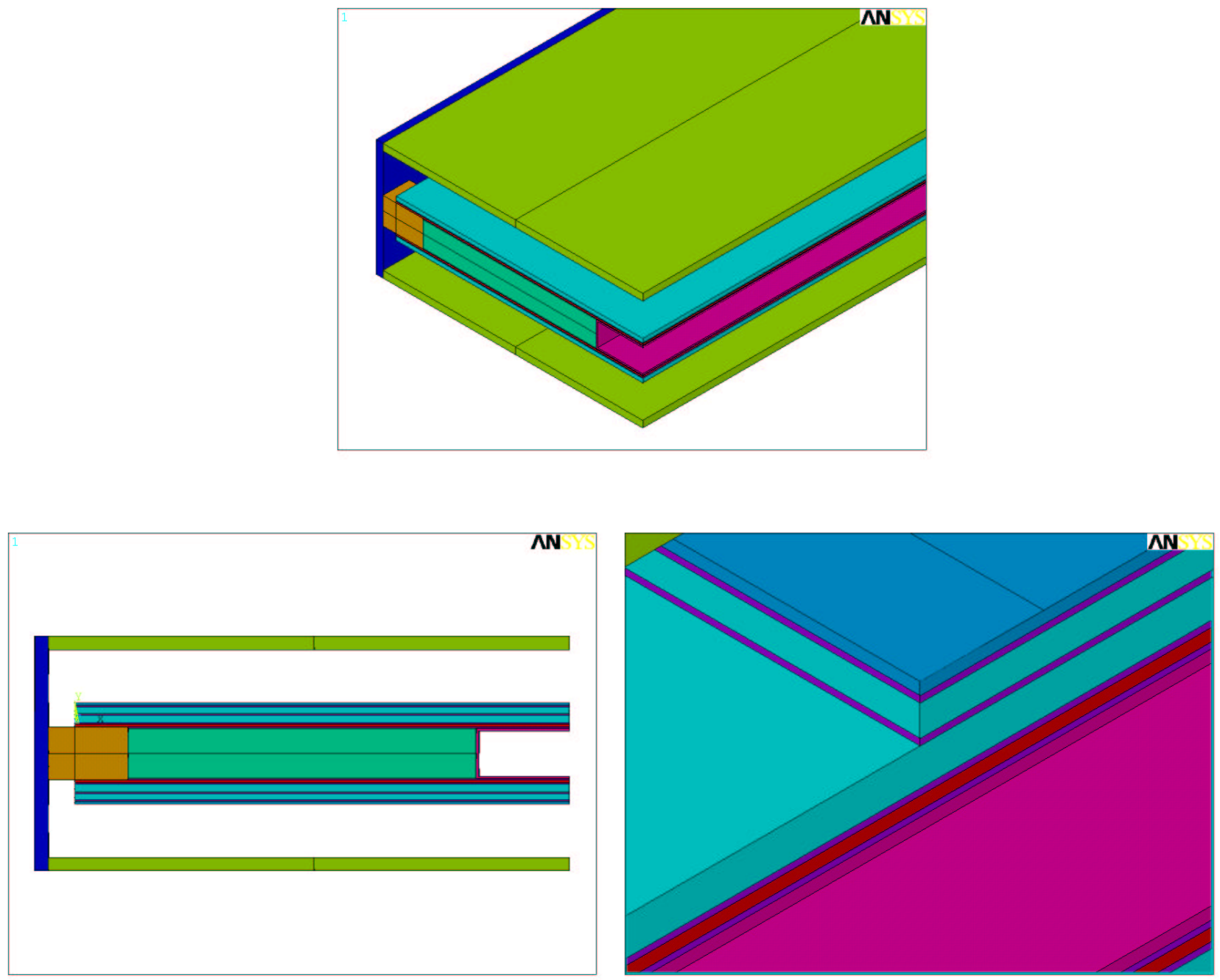

Figure 14 - Materials, isometric and front view of the detailed finite element model. Bottom right: silicon on-top mounted hybrid detail 
Fermi National Accelerator Laboratory

Giobatta Lanfranco Silicon Engineering Group - Mechanical Dep.

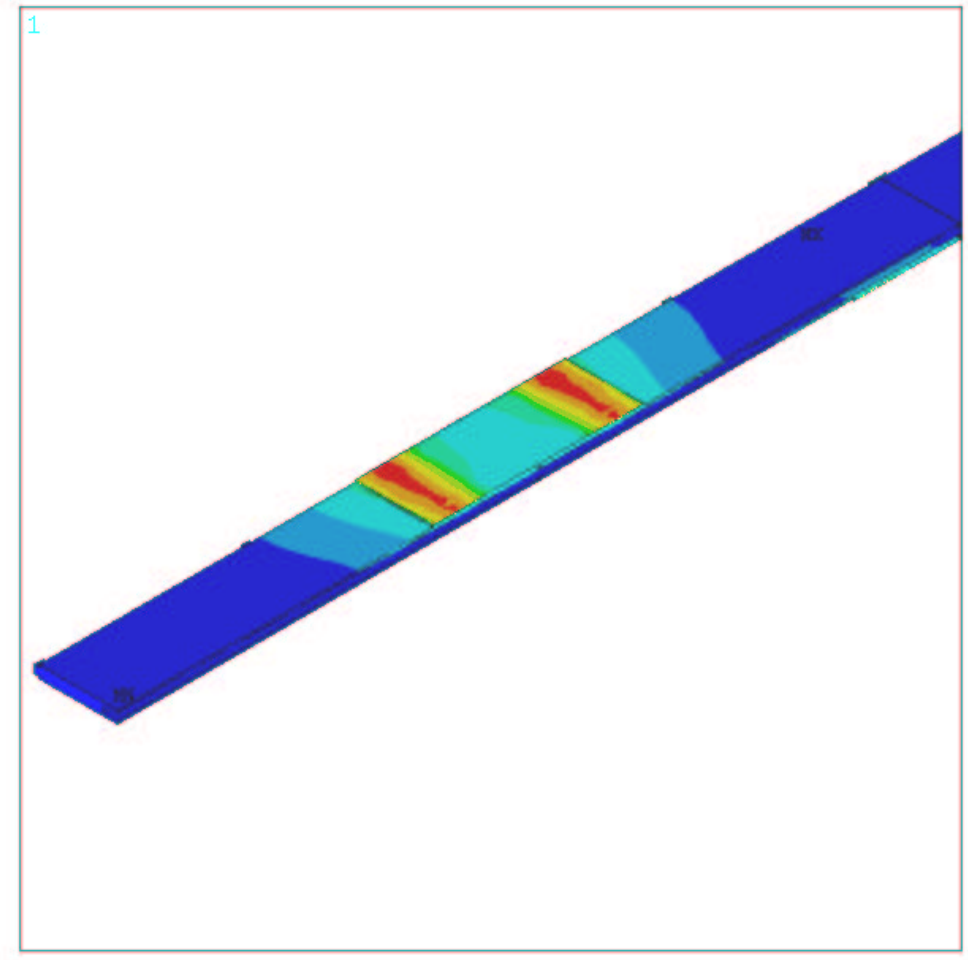

ANSYS 5.5 .3

JUN 282001

$16: 58: 26$

NODAL SOLUTION

$\mathrm{STEP}=1$

SUB $=1$
TIME $=1$

TEMP

SMN $=-9.973$

SMX $=14.148$

$-9.973$

$-7.293$

$-4.613$

$-1.933$

.747554

3. 428

6.108
8.788

11. 468

11.468
14.148

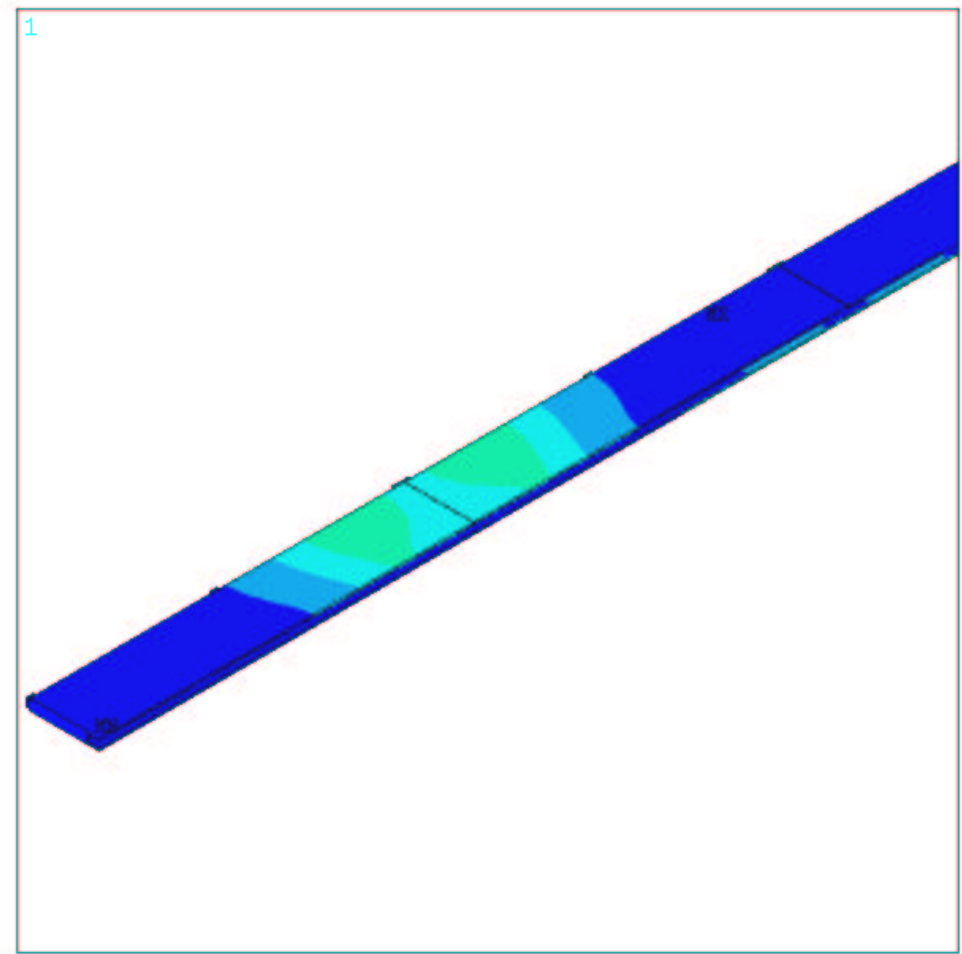

ANSYS 5.5 .3

JUN 282001

$16: 45: 09$

NODAL SOLUTION

STEP $=1$

SUB $=1$

T IME =1

TEMP

SMN $=-9.973$

SMX $=14.148$

SMX
-9.973

$-7.293$

-4.613
-1.933

.747554

3.428

6.108

8. 788

11. 468

14.148

Figure 15 - Temperature distribution along three ladders with staggered hybrids. Top: hybrids detail. Bottom: silicon detail. Half structure. 
Fermi National Accelerator Laboratory

Giobatta Lanfranco Silicon Engineering Group - Mechanical Dep.

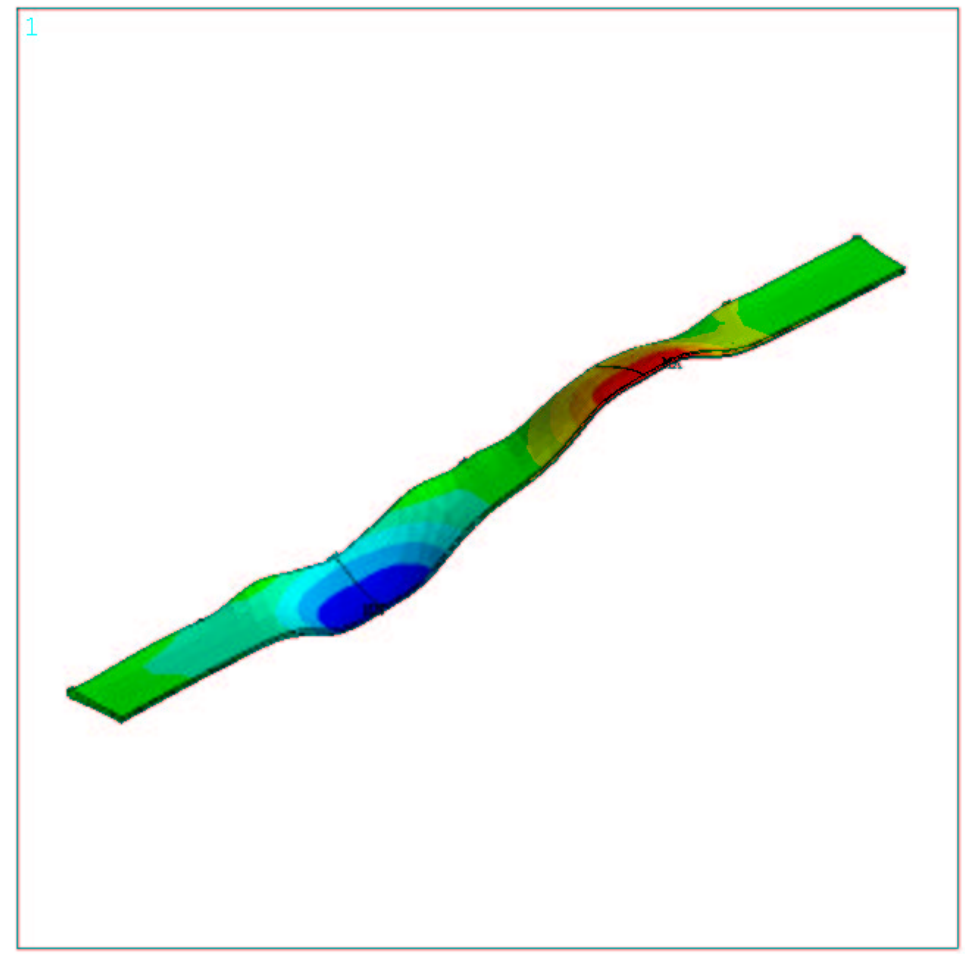

ANSYS $5.5 \cdot 3$

JUN 282001

$14: 01: 19$

NODAL SOLUTION

$\mathrm{STEP}=1$

SUB $=1$

TIME $=1$

UY

RSYS $=$ SOLU

$\mathrm{DMX}=.029637$

$\mathrm{SMN}=-.026725$

$\mathrm{SMX}=.029074$

$-.026725$

$-.020525$

$-.014326$

$-.008126$

$-.001926$

.004274

.010474

.016674

022874

.029074

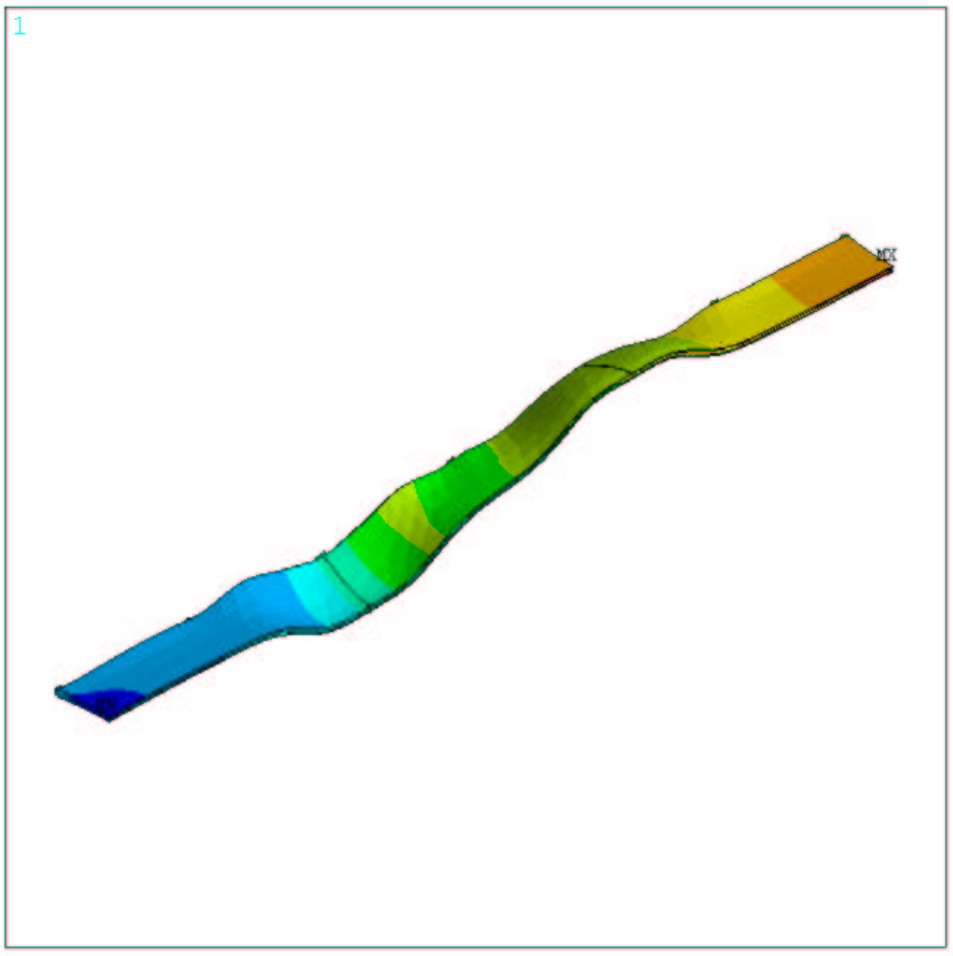

ANSYS 5.5 .3

JUN $28 \quad 2001$

$13: 52: 37$

NODAL SOLUTION

STEP $=1$

SUB $=1$

TIME $=1$

U2

RSYS $=S O L U$

$\mathrm{DMX}=.029637$

SMN $=-.002261$

$\mathrm{SMX}=.010048$

$-.002261$

$-.893 \mathrm{E}-03$

$.474 \mathrm{E}-03$

001842

.00321

004577

.005945

.007312

00868

.010048

Figure 16 - From top to bottom, vertical (UY) and longitudinal (UZ) displacement in silicon due to the temperature distribution given in Figure 15. Staggered hybrids. Half structure. 
Fermi National Accelerator Laboratory

Giobatta Lanfranco Silicon Engineering Group - Mechanical Dep.

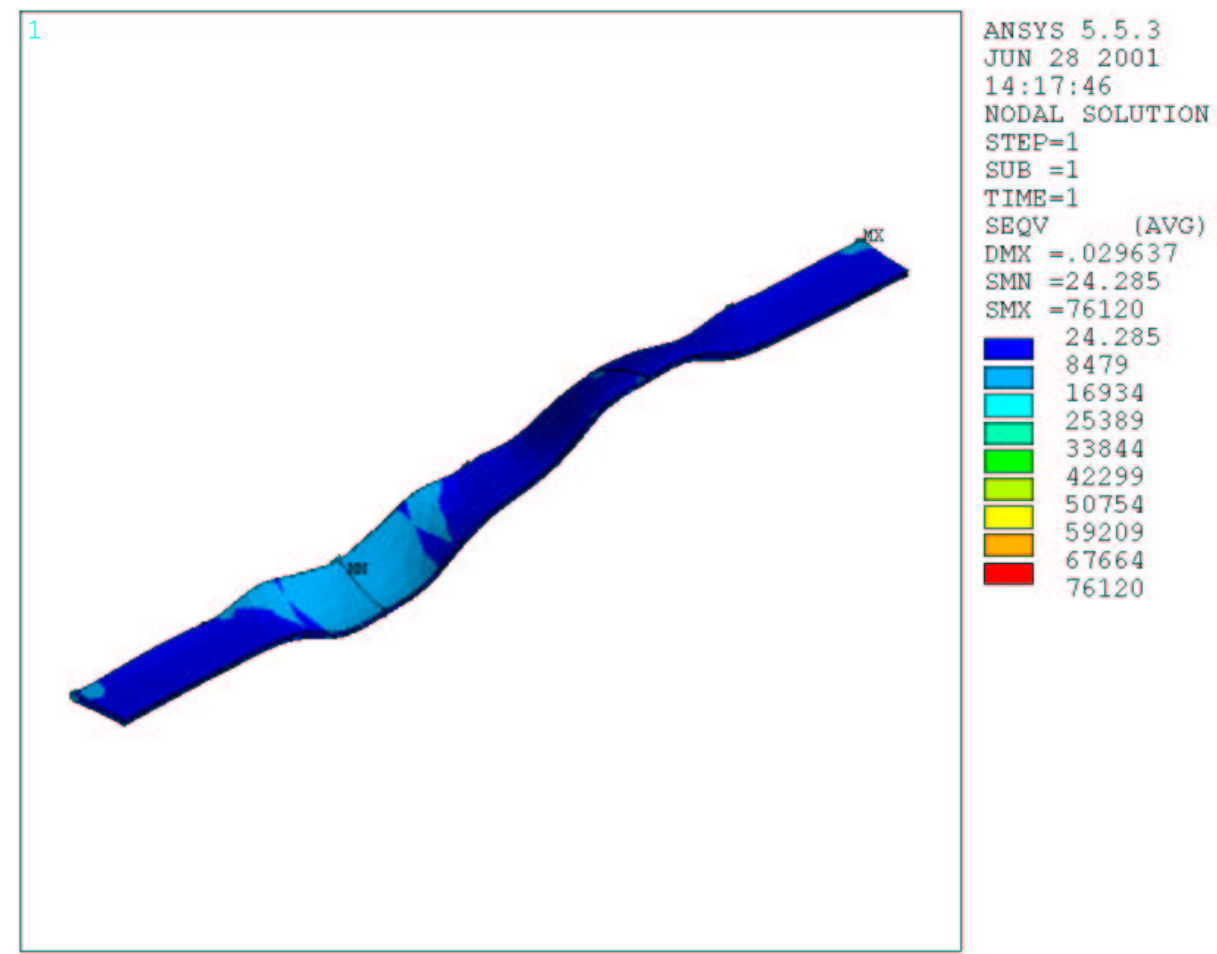

Figure 17 - Von Mises stress contour plot in silicon due to the temperature distribution given in Figure 15. Staggered hybrids. Half structure. [kPa, $1 / 7 \mathrm{psi}]$ 
Fermi National Accelerator Laboratory

Giobatta Lanfranco Silicon Engineering Group - Mechanical Dep.

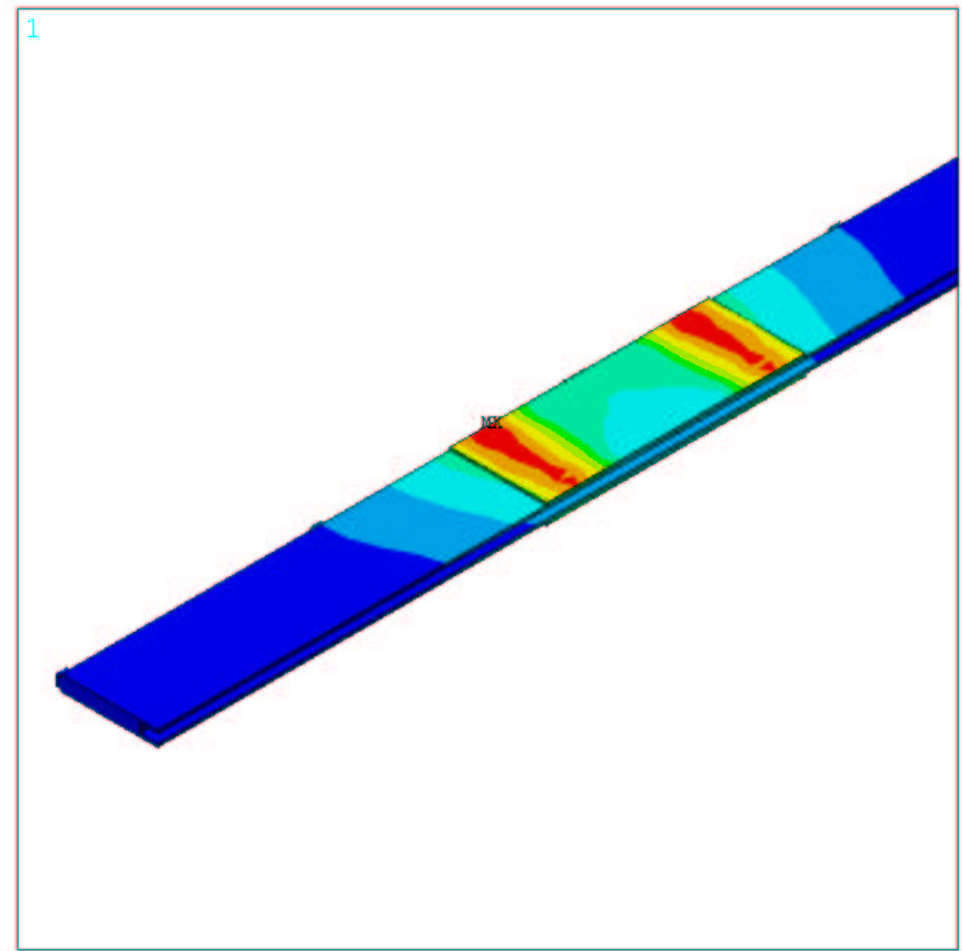

ANSYS $5 \cdot 5 \cdot 3$

JUN $28 \quad 2001$

$15: 02: 28$

NODAL SOLUTION

STEP $=1$

$\mathrm{SUB}=1$

TIME $=1$

TEMP

$\mathrm{SMN}=-9.996$

SMX $=14.74$

$-9.996$

$-7.248$

$-4.499$

$-1.751$

.997968

3. 746

6.495

9.243

11.992

14.74

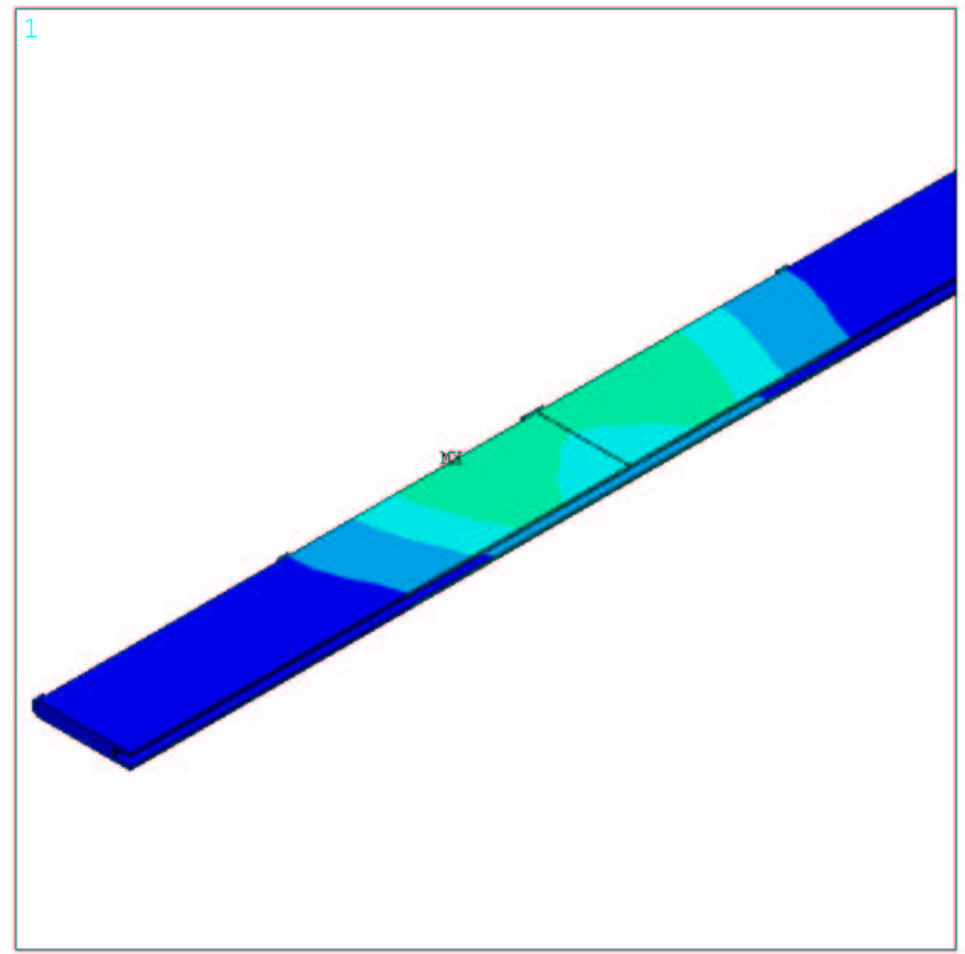

ANSYS $5 \cdot 5 \cdot 3$

JUN $28 \quad 2001$

$15: 03: 58$

NODAL SOLUTION

STEP $=1$

$B=$

TIME $=1$

TEMP

SMN $=-9.996$

SMX $=14.74$

-9.996
-

$-7.248$

$-4.499$

$-1.751$

.997968

3. 746

6.495

9.243

11.992

14.74

Figure 18 - Temperature distribution along three ladders with facing hybrids. Top: hybrids detail. Bottom: silicon detail. Half structure. 
Fermi National Accelerator Laboratory

Giobatta Lanfranco Silicon Engineering Group - Mechanical Dep.

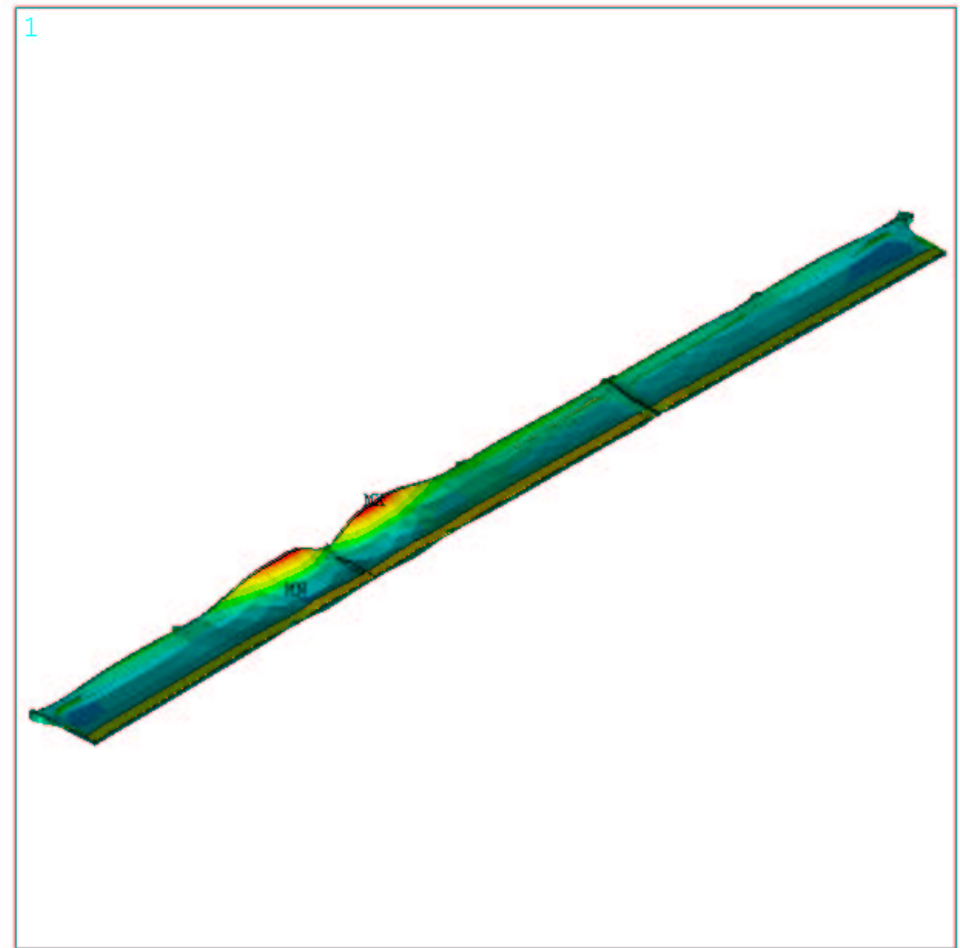

ANSYS $5.5 \cdot 3$

JUN $28 \quad 2001$

$16: 19: 36$

NODAL SOLUTION

STEP $=1$

SUB $=1$

TIME $=1$

UY

RSYS $=$ SOLU

DMX $=.009817$

SMN $=-.002815$

SMX $=, 004051$

$-.002815$

$-.002052$

$-.001289$

$-.526 \mathrm{E}-03$

$.237 E-03$

$.100 \mathrm{E}-02$

.001763

.002525

.003288

.004051

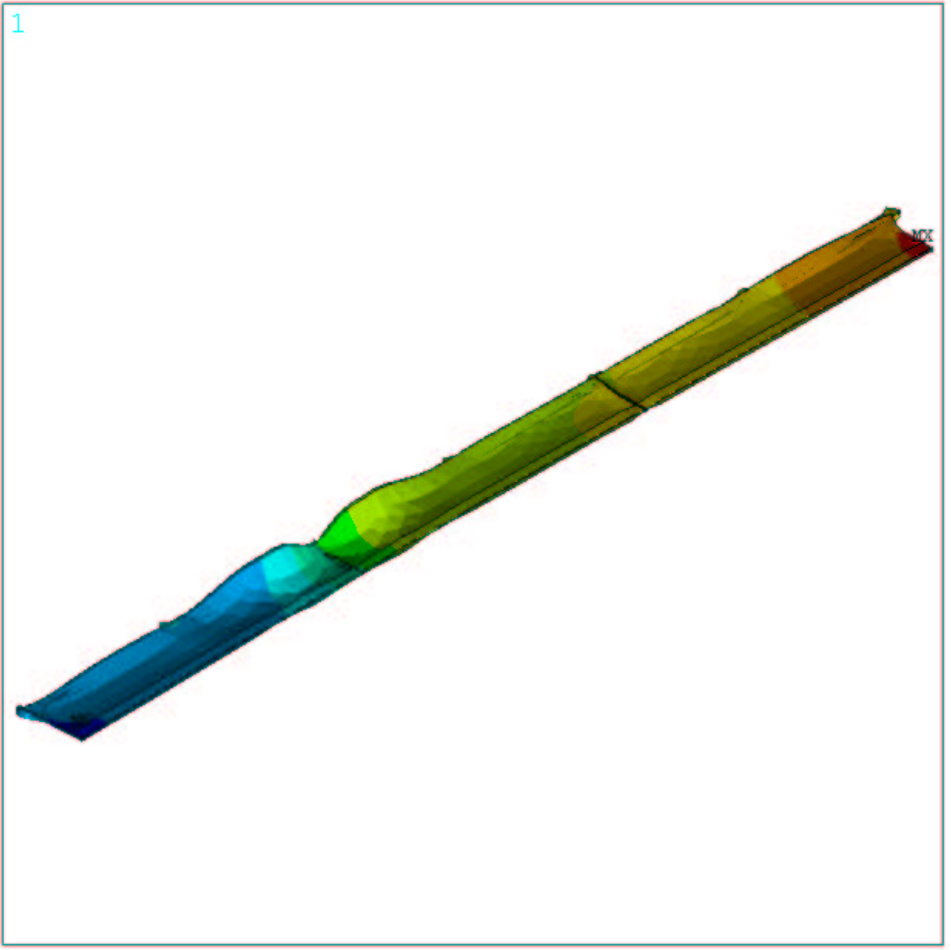

ANSYS 5.5 .3

JUN $28 \quad 2001$

$16: 17: 36$

NODAL SOLUTION

STEP $=1$

SUB $=1$

TIME $=1$

$\mathrm{UZ}$

RSYS $=$ SOLU

DMX $=.009817$

SMN $=-.002449$

SMX $=, 009809$

$-.002449$

$-.001087$

$.275 \mathrm{E}-03$

.001637

.002999

.004361

.005723

.007085

.008447

.009809

Figure 19 - From top to bottom, vertical (UY) and longitudinal (UZ) displacement in silicon due to the temperature distribution given in Figure 18. Facing hybrids. Half structure. 
Fermi National Accelerator Laboratory

Giobatta Lanfranco Silicon Engineering Group - Mechanical Dep.

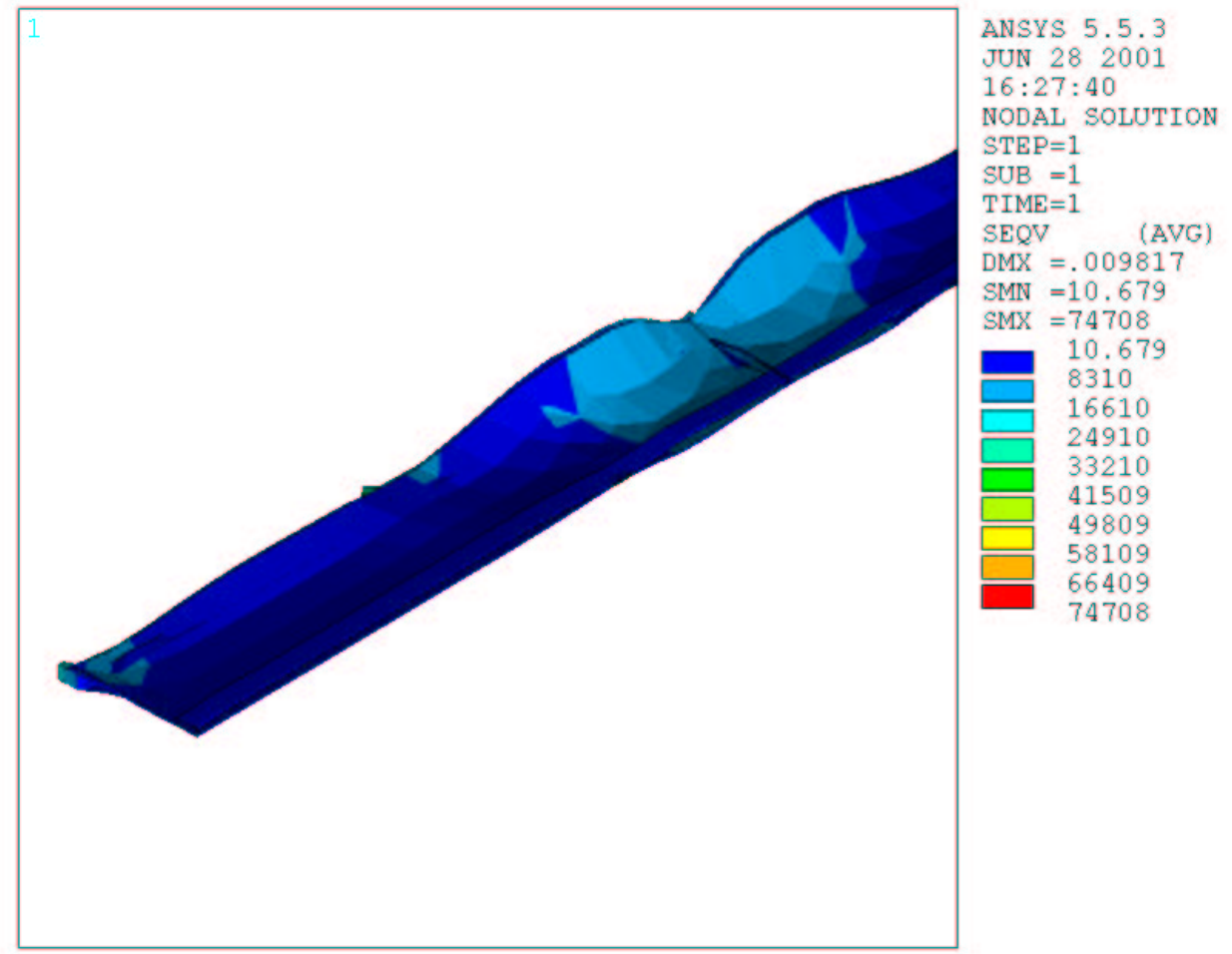

Figure 20 - Von Mises stress contour plot in silicon due to the temperature distribution given in Figure 18. Facing hybrids. Half structure. $[\mathrm{kPa}, \sim 1 / 7 \mathrm{psi}]$

\begin{tabular}{|c|c|c|c|c|c|c|c|c|}
\hline \multirow[t]{3}{*}{ case } & \multirow{3}{*}{$\begin{array}{c}\text { Chip } \\
\text { Max T } \\
{ }^{\circ} \mathrm{C}\end{array}$} & \multirow{3}{*}{$\begin{array}{c}\text { Silicon } \\
\text { Max T } \\
{ }^{\circ} \mathrm{C}\end{array}$} & \multicolumn{2}{|c|}{$\begin{array}{c}\text { Vertical } \\
\text { Displacement }\end{array}$} & \multicolumn{2}{|c|}{$\begin{array}{l}\text { Longitudinal } \\
\text { Displacement }\end{array}$} & \multicolumn{2}{|c|}{$\begin{array}{c}\text { Stress } \\
\text { (Von Mises) }\end{array}$} \\
\hline & & & $\min$ & $\max$ & $\min$ & $\max$ & $\min$ & $\max$ \\
\hline & & & $\mu \mathrm{m}$ & $\mu \mathrm{m}$ & $\mu \mathrm{m}$ & $\mu \mathrm{m}$ & $\mathrm{MPa}$ & $\mathrm{MPa}$ \\
\hline $\mathrm{I}$ & 14.1 & $\sim 0$ & -26 & 29 & -2 & 10 & 0.024 & 76.120 \\
\hline II & 14.7 & 1 & -3 & 4 & -2 & 10 & 0.010 & 74.708 \\
\hline
\end{tabular}

Table 8 - Single channel staves analysis summarize

A comparison with a double cooling channel stave is reported in the following pictures and the results are summarized in Table 9. Although the structure has poorer mechanical performance, it may be preferable on the thermal standpoint, providing a considerable lower working temperature in the chip. 
Fermi National Accelerator Laboratory

Giobatta Lanfranco Silicon Engineering Group - Mechanical Dep.
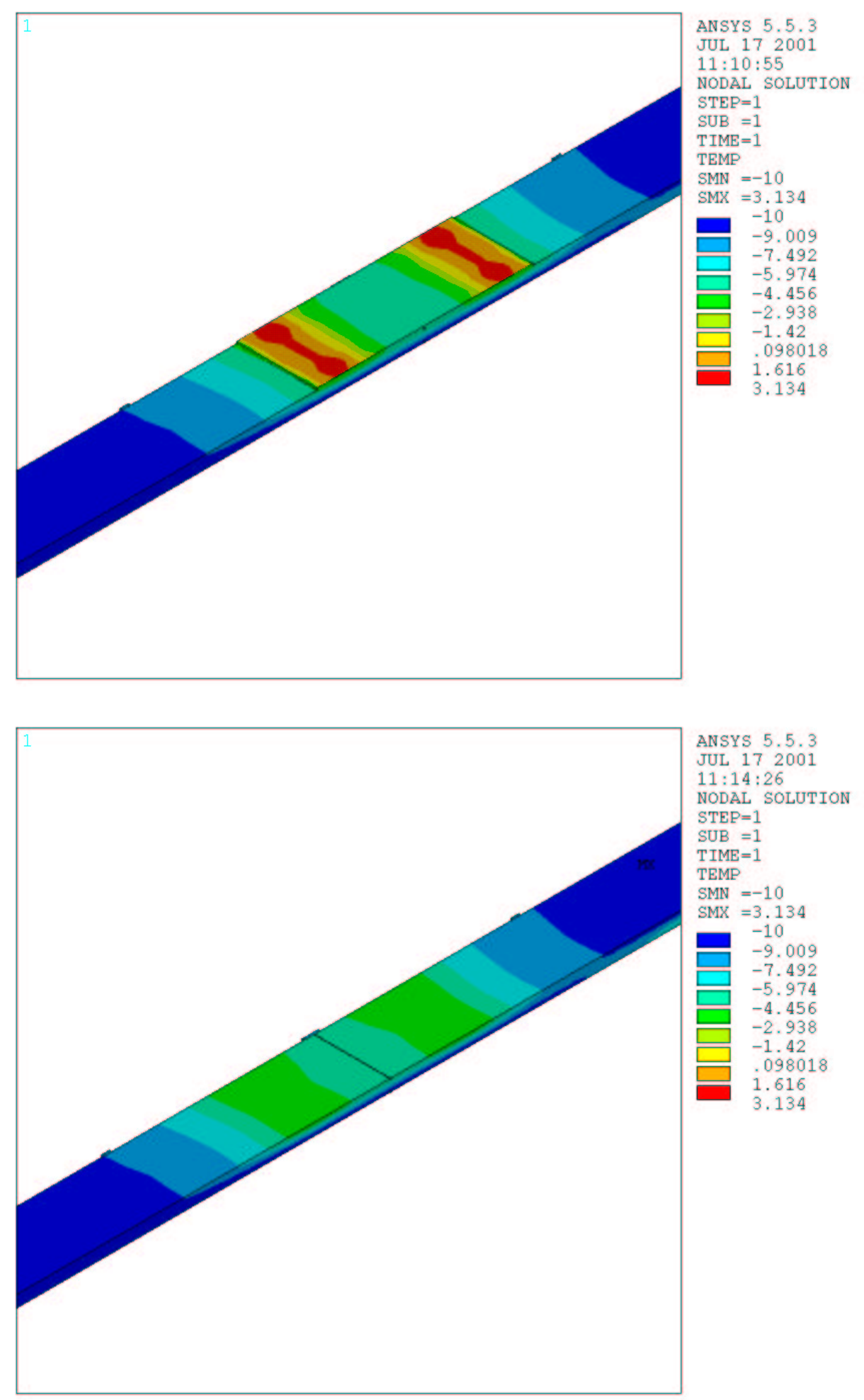

Figure 21 - Temperature distribution along three ladders with staggered hybrids. Top: hybrids detail. Bottom: silicon detail. Half structure. DOUBLE CHANNEL COOLING 
Fermi National Accelerator Laboratory

Giobatta Lanfranco Silicon Engineering Group - Mechanical Dep.

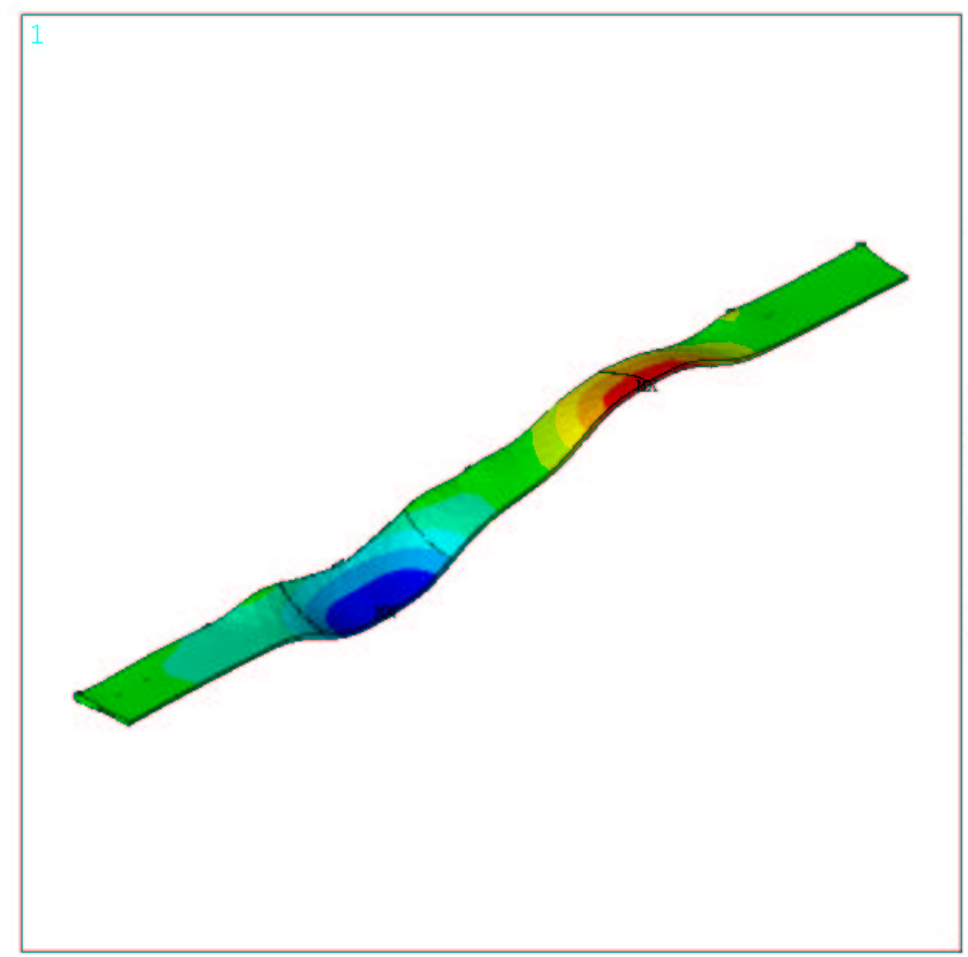

ANSYS 5.5 .3

JUL 17.2001

$15: 36: 13$

NODAL SOLUTION

STEP $=1$

SUB $=1$

TIME $=1$

UY

RSYS $=$ SOLU

DMX $=.034327$

SMN $=-.031978$

$\operatorname{SMX}=, 033583$

$-.031978$

$-.024694$

$-.017409$

$-.010125$

$-.00284$

.004445

.011729

.019014

.026298

033583

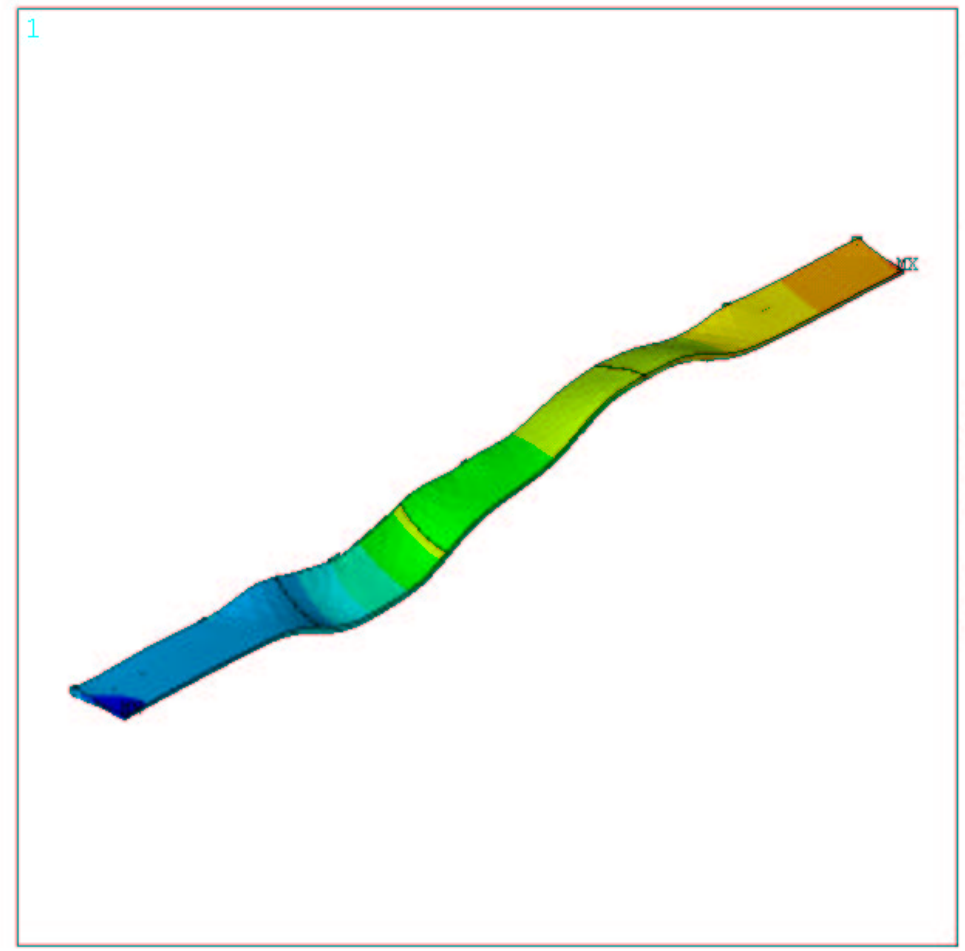

ANSYS 5.5 .3

JUL 172001

$15: 37: 00$

NODAL SOLUTION

STEP $=1$

SUB $=1$

TIME $=1$

$\mathrm{Uz}$

RSYS $=$ SOLU

$\mathrm{DMX}=.034327$

SMN $=-.003347$

SMX $=.014546$

$-.003347$

$-.001359$

$.629 \mathrm{E}-03$

002617

.004605

.006593

008581

010569

.012557

.014546

Figure 22 - From top to bottom, vertical (UY) and longitudinal (UZ) displacement in silicon due to the temperature distribution given in Figure 21. Staggered hybrids. Half structure. DOUBLE CHANNEL COOLING 
Fermi National Accelerator Laboratory

Giobatta Lanfranco Silicon Engineering Group - Mechanical Dep.

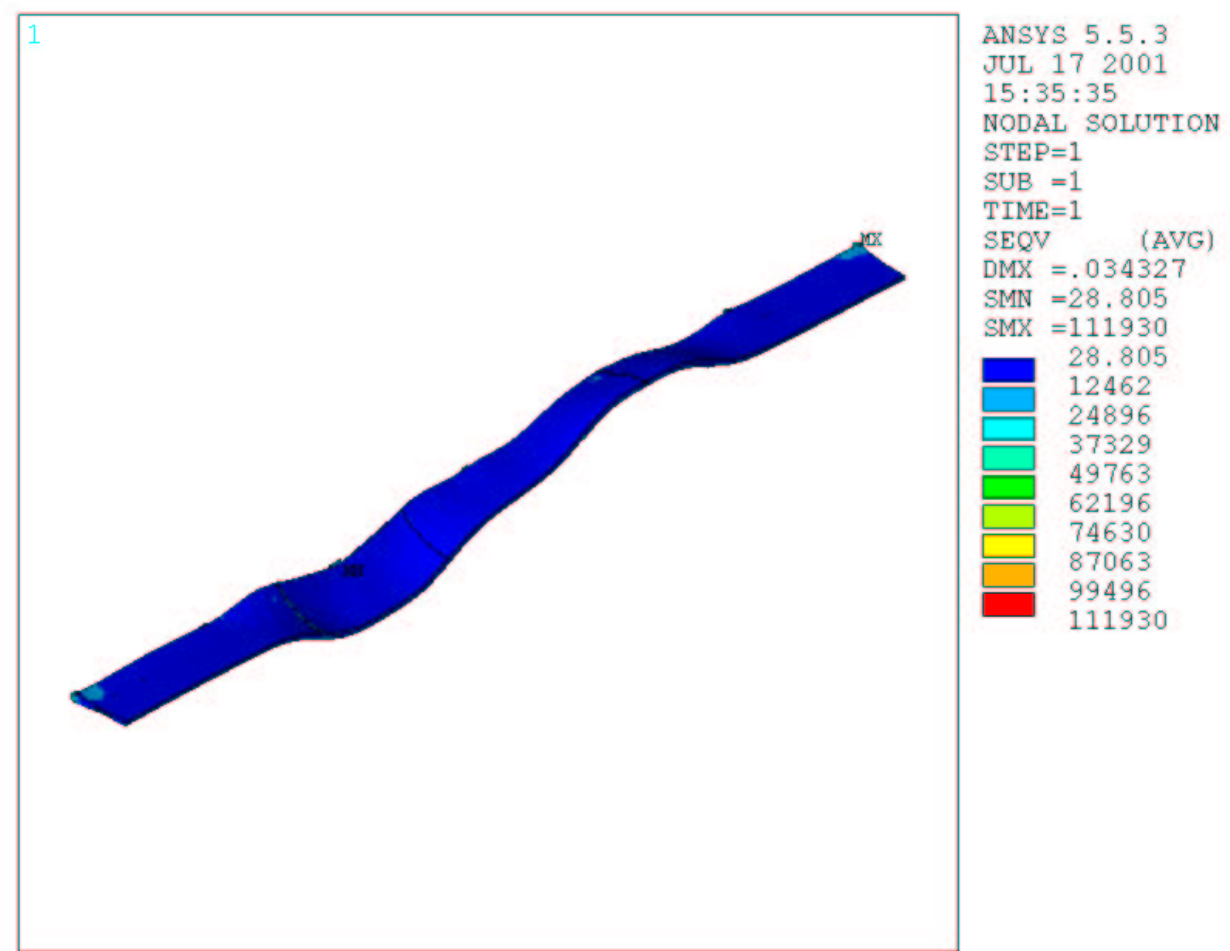

Figure 23 - Von Mises stress contour plot in silicon due to the temperature distribution given in Figure 21. Staggered hybrids. Half structure. [kPa, 1/7 psi]. DOUBLE CHANNEL COOLING

\begin{tabular}{|c|c|c|cc|cc|cc||}
\hline \hline \multirow{2}{*}{ case } & \multirow{2}{*}{ Chip } & Silicon & \multicolumn{2}{|c|}{ Vertical } & \multicolumn{2}{c|}{ Longitudinal } & \multicolumn{2}{c|}{ Stress } \\
& Max T & Max T & \multicolumn{2}{|c|}{ Displacement } & \multicolumn{2}{c|}{ Displacement } & \multicolumn{2}{c|}{ (Von Mises) } \\
& & & \multicolumn{2}{c|}{$\min$} & $\max$ & $\min$ & $\max$ & \multicolumn{2}{c|}{$\min$} & $\max$ \\
& ${ }^{\circ} \mathrm{C}$ & ${ }^{\circ} \mathrm{C}$ & $\mu \mathrm{m}$ & $\mu \mathrm{m}$ & $\mu \mathrm{m}$ & $\mu \mathrm{m}$ & $\mathrm{MPa}$ & $\mathrm{MPa}$ \\
\hline $\mathrm{I}$ & 3.1 & $\sim-2$ & -30 & 33 & -3 & 14 & 0.028 & 112 \\
\hline
\end{tabular}

Table 9 - Double channel staves analysis summarize

\section{Conclusions}

Problems related to the coefficient of thermal expansion mismatches may cause dangerous stress buildups in the silicon sensor as well as compromise its micrometric alignment with the beam. The use of a high conductive carbon fiber heat spreader, along with a Rohacell core, may effectively help preventing the thermal bowing; at the same time, restraining the sensor from longitudinal contraction, with high modulus carbon fiber shell that does not match the silicon CTE, does not introduce dangerous stress peaks. 
Fermi National Accelerator Laboratory

Giobatta Lanfranco Silicon Engineering Group - Mechanical Dep.

For the problems related to meshing a thin shell, that is poor quality elements, a further experimental study is recommended. 